\title{
The mean star-forming properties of QSO host galaxies
}

\author{
D. J. Rosario ${ }^{1}$, B. Trakhtenbrot ${ }^{2}$, D. Lutz ${ }^{1}$, H. Netzer ${ }^{3}$, J. R. Trump ${ }^{4}$, J. D. Silverman ${ }^{5}$, M. Schramm ${ }^{5}$, E. Lusso ${ }^{6}$,
} S. Berta ${ }^{1}$, A. Bongiorno ${ }^{7}$, M. Brusa ${ }^{8}$, N. M. Förster-Schreiber ${ }^{1}$, R. Genzel ${ }^{1}$, S. Lilly ${ }^{9}$, B. Magnelli ${ }^{10}$, V. Mainieri ${ }^{11}$, R. Maiolino ${ }^{12,13}$, A. Merloni ${ }^{1}$, M. Mignoli ${ }^{14}$, R. Nordon $^{1}$, P. Popesso ${ }^{1}$, M. Salvato ${ }^{1}$, P. Santini ${ }^{7}$, L. J. Tacconi ${ }^{1}$, and G. Zamorani ${ }^{14}$

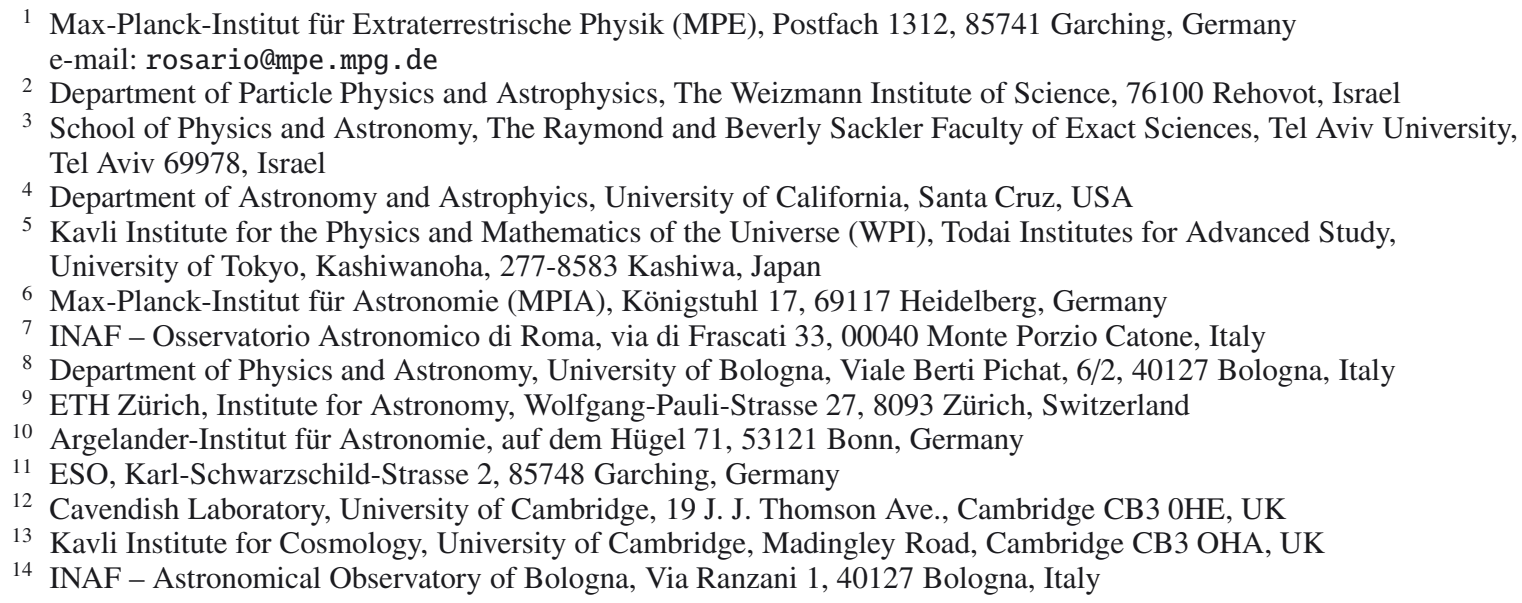

Received 2 July 2013 / Accepted 6 October 2013

\section{ABSTRACT}

\begin{abstract}
Quasi-stellar objects (QSOs) occur in galaxies in which supermassive black holes (SMBHs) are growing substantially through rapid accretion of gas. Many popular models of the co-evolutionary growth of galaxies and black holes predict that QSOs are also sites of substantial recent star formation (SF), mediated by important processes, such as major mergers, which rapidly transform the nature of galaxies. A detailed study of the star-forming properties of QSOs is a critical test of these models. We present a far-infrared Herschel/PACS study of the mean star formation rate (SFR) of a sample of spectroscopically observed QSOs to $z \sim 2$ from the COSMOS extragalactic survey. This is the largest sample to date of moderately luminous QSOs (with nuclear luminosities that lie around the knee of the luminosity function) studied using uniform, deep far-infrared photometry. We study trends of the mean SFR with redshift, black hole mass, nuclear bolometric luminosity, and specific accretion rate (Eddington ratio). To minimize systematics, we have undertaken a uniform determination of SMBH properties, as well as an analysis of important selection effects of spectroscopic QSO samples that influence the interpretation of SFR trends. We find that the mean SFRs of these QSOs are consistent with those of normal massive star-forming galaxies with a fixed scaling between SMBH and galaxy mass at all redshifts. No strong enhancement in SFR is found even among the most rapidly accreting systems, at odds with several co-evolutionary models. Finally, we consider the qualitative effects on mean SFR trends from different assumptions about the SF properties of QSO hosts and from redshift evolution of the SMBH-galaxy relationship. While currently limited by uncertainties, valuable constraints on AGN-galaxy co-evolution can emerge from our approach.
\end{abstract}

Key words. surveys - galaxies: active - galaxies: star formation - quasars: general - galaxies: high-redshift - infrared: galaxies

\section{Introduction}

Quasi-stellar objects (QSOs) constitute the luminous end of the population of broad-line AGNs (BLAGNs), i.e., those that display broad permitted and semi-forbidden emission lines in their spectra with full width at half maximum (FWHM) of few to several thousands of $\mathrm{km} \mathrm{s}^{-1}$. The luminosity of QSOs - they heavily outshine their host galaxies, especially at ultra-violet (UV) and optical wavelengths - allow them to be detected at very large cosmological distances, and the low intrinsic obscuration they exhibit towards the nuclear engine make them the principal laboratories used by researchers for understanding AGN accretion, environments, and energetics.
The widespread existence of supermassive black holes (SMBHs) in local galaxies and the tight relationships they exhibit with respect to the masses of their host galaxies (e.g., Magorrian et al. 1998; Tremaine et al. 2002; Graham \& Driver 2007; Aller \& Richstone 2007) suggest a close relationship between the stellar growth of galaxies and the phases of maximal growth of black holes. This has stimulated much study into the co-evolutionary relationship between galaxies and AGNs. Most of the cosmic growth of SMBHs takes place at $z=$ 1-2 in luminous AGNs with bolometric nuclear luminosities $L_{\text {bol }}>10^{45} \mathrm{erg} \mathrm{s}^{-1}$ (Page et al. 2004). QSOs are the primary tracer of this population, though some studies suggest that much black hole growth may also occur in obscured phases that are 
missed in traditional QSO samples (e.g., Martínez-Sansigre et al. 2005; Polletta et al. 2006; Donley et al. 2007; Reyes et al. 2008) or through X-ray selection (e.g., Daddi et al. 2007a; Gilli et al. 2007; Fiore et al. 2008, 2009; Alexander et al. 2011). In spite of this, almost all models of AGN-galaxy co-evolution ascribe a special role to the QSO population. For example, the popular evolutionary scenario that links elliptical galaxies to gas-rich major mergers through a massive starburst predicts a brief period of luminous AGN activity that is eventually visible as an optically bright QSO (Sanders et al. 1988; Granato et al. 2004; Hopkins et al. 2008). An important corollary is that QSOs should be associated with the sites of current or post-starbursts. The exact relationship between QSOs and starbursts depends on the nature and timing of the poorly constrained luminous obscured AGN phase believed to exist before strong feedback clears out the dust and gas from the merger remnant. However, a close correspondence between QSOs and recent starburst events is predicted even in co-evolutionary models that do not explicitly rely on major galaxy mergers to fuel QSOs (e.g., Ciotti \& Ostriker 2007).

Star formation (SF) in QSO host galaxies has been extensively studied using high-resolution imaging in the optical (e.g., Bahcall et al. 1997; Dunlop et al. 2003; Jahnke et al. 2004) and near-infared (e.g., Kukula et al. 2001; Guyon et al. 2006; Veilleux et al. 2009); emission line tracers such as the [O II] line (Hes et al. 1993; Ho 2005; Silverman et al. 2009; Kalfountzou et al. 2012); mid-infrared emission lines and PAH features (Netzer et al. 2007; Lutz et al. 2008; Shi et al. 2009); and farinfrared (FIR) and sub-mm photometry (e.g., Priddey et al. 2003; Omont et al. 2003; Lutz et al. 2010; Serjeant \& Hatziminaoglou 2009; Serjeant et al. 2010; Bonfield et al. 2011). In general, QSO hosts are in massive, spheroidally-dominated galaxies, which frequently show signs of on-going star-formation (e.g., Jahnke et al. 2004; Trump et al. 2013), though signatures of early stage mergers or strong disturbances are not particularly frequent (Dunlop et al. 2003; Guyon et al. 2006; Bennert et al. 2008; Veilleux et al. 2009). Very powerful starbursts are known to exist among high redshift QSOs, with $\sim 30 \%$ of very optically luminous systems showing star formation rates (SFRs) at the level of thousands of $M_{\odot} / \mathrm{yr}$ at $z \sim 2$ (e.g., Omont et al. 2003; Wang et al. 2008). Additional evidence from CO and [C II] observations indicate large gas supplies that could fuel starbursts (e.g., Walter et al. 2004, 2009; Coppin et al. 2008; Wang et al. 2010, 2013). However, it is clear that not all QSOs are in strong starbursts - local PGQSOs span SFRs ranging from very low to $\sim 100 M_{\odot} /$ yr (Schweitzer et al. 2006; Netzer et al. 2007). Studies of high-redshift luminous Type II AGNs, obscured counterparts of QSOs, suggest typically modest SFRs comparable to normal SF galaxies (Sturm et al. 2006; Mainieri et al. 2011).

Understanding the link between strong bursts of SF and QSO activity is complicated by a few important biases. Firstly, bright QSOs are essentially all in very massive galaxies. For an evaluation of whether QSOs are indeed in galaxies with abnormally high levels of SF, a proper comparison has to be made with the SFRs of inactive galaxies at the same redshifts and of similar stellar mass, since SFR is strongly correlated both with redshift and stellar mass among SF galaxies (Noeske et al. 2007; Elbaz et al. 2007; Daddi et al. 2007b; Wuyts et al. 2011; Whitaker et al. 2012). Secondly, BLAGN populations are essentially defined by spectroscopic surveys, which have important selection effects that must be taken into account when statistically evaluating black hole and host galaxy properties (Shen \& Kelly 2012, and Sect. 4.1). In this study, we explore the SF properties of QSO hosts, relying on the FIR as a relatively clean measure of the total luminosity of SF-heated dust (Netzer et al. 2007; Rosario et al. 2012). We start by compiling one of the largest samples of broad-line AGNs in a deep extragalactic survey field with uniformly measured SMBH properties. The combination of sample size, redshift coverage, and spectroscopic and FIR imaging depth is unsurpassed in existing studies of distant QSOs. From this compilation, we determine the mean SFRs of moderately luminous QSOs through the stacking of Herschel/PACS images, while using simple models to account for the effects of sample biases and explore the relationship between SMBH growth and global star-formation.

As the sample consists of fairly luminous systems, we use the term QSOs or BLAGNs to refer to all broad-line AGNs throughout this paper. Additionally, in Sect. 5.1, we compare our QSOs with AGNs selected using X-rays, which comprise a larger and more diverse set of objects. These X-ray AGNs encompass broad-line, narrow-line, or optically dull AGNs.

We assume a standard $\Lambda$-CDM Concordance cosmology, with $H_{0}=70 \mathrm{~km} \mathrm{~s}^{-1} \mathrm{Mpc}^{-1}$ and $\Omega_{\Lambda}=0.7$. Stellar masses in this study, where reported, assume a Chabrier initial mass function (Chabrier 2003).

\section{Datasets and sample selection}

\subsection{Selection of broad-line AGNs}

For a substantial sample of BLAGNs over a range of redshifts with associated deep FIR and X-ray imaging coverage, we concentrated on the $2 \mathrm{deg}^{2}$ medium-deep COSMOS extragalactic survey field (Scoville et al. 2007). This field has been the target of multiple optical spectroscopic surveys of varying depths and we turn to several of these datasets to select BLAGNs. In practice, the size of the sample is restricted by the redshifts at which broad $\mathrm{H} \beta$ and MgII $\lambda 2800$ lines can be reliably measured in optical spectra, as well as the capabilities of the broad-line fitting method used to derive SMBH masses. For example, several sources were excluded after a manual inspection of their fits, because they suffered from bad data, were too close to the edge of a spectrum, or were simply limited by the signal-to-noise ratio $(\mathrm{S} / \mathrm{N})$ of the spectra.

The Sloan Digital Sky Survey (SDSS) DR7 spectroscopic database (Abazajian et al. 2009), which covers the COSMOS field, was used to select a subset of QSOs. Targets with high quality redshifts and marked by the class "QSO" were identified, from which radio-loud sources (based on $20 \mathrm{~cm}$ fluxes in the FIRST survey) and broad absorption line systems (BALs) were removed. Details of the selection method can be found in Trakhtenbrot \& Netzer (2012). Our total SDSS subsample consists of 70 BLAGNs with measurable SMBH masses.

The zCOSMOS survey (Lilly et al. 2007, 2009) is a multi-purpose spectroscopic campaign in COSMOS that uses the VIMOS spectrograph on the VLT. It consists of two tiers: zCOSMOS-Bright targets 20000 galaxies over the entire COSMOS/ACS field to $I_{A B}=22.5$, while zCOSMOS-Deep targets 10000 galaxies over the inner $1 \mathrm{deg}^{2}$ of the field to a deeper limit of $B_{A B}=25$ with an additional color-based galaxy preselection. From both tiers, QSOs were classified based on spectral features and an automated comparison to a QSO template, followed by visual assessment of the fit. Only objects with the Mg II $\lambda 2800$ line were included in our sample. The zCOSMOS survey yields a total of 176 BLAGNs with measurable SMBH masses, 146 from the Bright survey and 30 from the Deep survey. 
The extensive X-ray point source catalog in the COSMOS field (Brusa et al. 2010) from the XMM-COSMOS X-ray survey (Cappelluti et al. 2009) has been the basis of spectroscopic follow-up programs. In addition to the SDSS and zCOSMOS sources described above, we incorporated a sample of BLAGNs selected from a Magellan/IMACS program of X-ray source follow-up (Trump et al. 2007, 2009a) which yielded 112 BLAGNs for which we could measure SMBH masses. Nominal flux limits for this dataset are $i_{A B}^{+}=23.5$, sampling fainter sources than zCOSMOS-Bright.

Combining the three subsamples and resolving duplicates (i.e, the same AGN observed in two or more spectroscopic surveys), we arrived at a final QSO working sample of 289 objects. This is currently the largest sample of BLAGNs with deep, uniform FIR coverage and reliably estimated SMBH properties.

\subsection{Herschel imaging and photometry}

The FIR data used in this work were collected by the PACS instrument (Poglitsch et al. 2010) on board the Herschel Space Observatory, as part of the PACS Evolutionary Probe (PEP, Lutz et al. 2011) survey. Observations of almost the entire COSMOS field were taken in two PACS bands (100 and $160 \mu \mathrm{m})$. We make use of PACS catalogs extracted using the prior positions and fluxes of sources detected in deep Spitzer/MIPS $24 \mu \mathrm{m}$ imaging in the field (Le Floc'h et al. 2009). This allows us to accurately deblend PACS sources in images characterized by a large point spread function (PSF), especially in crowded fields, and greatly improve the completeness of faint sources at the detection limit. The $3 \sigma$ limits of the PACS catalogs are 5.0/11.0 mJy at $100 / 160 \mu \mathrm{m}$. We consider sources below these limits to be undetected by PACS. Residual maps, from which all detected sources are subtracted, were used in the stacking procedure described in Sect. 3.2. Detailed information on the PEP survey, observed fields, data processing and source extraction can be found in Lutz et al. (2011).

Of the 272 QSOs that lie within the region of uniform FIR coverage, 38 (14\%) were detected in both PACS bands. This is slightly higher than the FIR detection rate of $10 \%$ among more luminous QSOs presented in Dai et al. (2012) from somewhat shallower Herschel/SPIRE imaging. Given that their bolometric luminosity and redshift ranges are different from ours, we refrain from a detailed comparison of the two samples, but note that the rough consistency in the detection rates suggests that the FIR luminosity of QSOs does not rise dramatically with nuclear luminosity.

\subsection{X-ray photometry}

We crossmatched our working sample to the optical counterparts of X-ray point sources from the XMM-COSMOS survey (Cappelluti et al. 2009; Brusa et al. 2010). There are 243 BLAGNs that have an associated X-ray point source, which is $86 \%$ of the sample. Of the remaining 39 sources with no X-ray counterpart, a fraction lie on the edges of the XMM-COSMOS field, where the X-ray depths are shallower than in the center of the field. However, there are a few genuine BLAGNs that have no X-ray counterparts even at the center of the XMM-COSMOS field. These are examples of relatively rare X-ray faint but optically luminous AGNs (Vignali et al. 2001).

We make use of absorption-corrected rest-frame X-ray luminosities in the hard band $\left(2-10 \mathrm{keV} ; L_{\mathrm{X}}\right.$ hereafter) for X-ray AGNs and QSOs with X-ray detections. Details of the estimation of $L_{\mathrm{X}}$, as well as information about redshifts and other properties of the XMM-COSMOS catalog used here, can be found in Santini et al. (2012) and Rosario et al. (2012).

\section{Methods}

\subsection{SMBH masses and bolometric luminosities}

Before embarking on the measurement of SMBH masses, we checked the relative spectrophotometric performance of the zCOSMOS and IMACS datasets by comparing the spectra of a set of objects that overlapped between these two samples. We found systematic flux offsets between the spectra at the level of $\approx 0.4$ dex. The comparison of zCOSMOS and SDSS spectra of a small set of overlapping objects also suggested an offset of $\approx 0.3$ dex. The IMACS and zCOSMOS spectra are subject to a seeing-dependent slit-loss due to the $1^{\prime \prime}$ width of the slit (Trump et al. 2009a; Merloni et al. 2010), which could be the cause of most of the observed offsets.

To account for these remaining spectrophotometric offsets, we measured synthetic broad-band magnitudes directly from the zCOSMOS and IMACS spectra and compared them to integrated broad-band photometry of the QSOs in the public COSMOS multiwavelength catalog (Capak et al. 2007). The differences were used to estimate correction factors that were then applied to the spectra. The zCOSMOS-Bright and IMACS spectra, which cover the approximate wavelength range of 5500 $9500 \AA$, were scaled to CFHT/Megaprime i* photometry, while the zCOSMOS-Deep spectra, with wavelength coverage from 3600-6800 A, were scaled to Subaru/Suprime-Cam g+ photometry. After applying the scaling factors, a visual comparison of the spectra of overlapping objects showed broad consistency in both the continuum normalization and the shape of the spectra.

Black hole masses $\left(M_{\mathrm{BH}}\right)$ were estimated using virial relationships calibrated from the reverberation mapping of local BLAGNs, following the methodology detailed in Trakhtenbrot $\&$ Netzer (2012). We used only virial relationships for the broad $\mathrm{H} \beta$ and MgII $\lambda 2800$ emission lines, which effectively restricts the redshifts probed by our sample to $z<2.2$. It has been proposed that, in principle, the broad CIV $\lambda 1550$ line can be used to estimate $M_{\mathrm{BH}}$ at higher redshifts. However, several studies have shown that such estimates are highly unreliable (see Trakhtenbrot \& Netzer 2012, and references therein), and we therefore exclude CIV-based masses in this work.

Our fitting method is detailed in Trakhtenbrot \& Netzer (2012). Broad lines are modeled as combinations of broad and narrow Gaussian components fit along with the underlying continuum, narrow absorption features, and with an optimised template to account for FeII and FeIII band emission. All the fits were visually inspected and vetted, while a fraction were improved manually.

Bolometric luminosities $\left(L_{\mathrm{bol}}\right)$ of the AGNs were estimated using bolometric corrections to the monochromatic luminosities at either $5100 \AA$ or $3000 \AA$ rest-frame. The choices of bolometric corrections are derived in Trakhtenbrot \& Netzer (2012) and are consistent with the prescriptions of Marconi et al. (2004), though slightly lower than some other commonly used values (e.g., Richards et al. 2006).

We calibrate the performance and uncertainties on our measurements by comparing $M_{\mathrm{BH}}$ and $L_{\mathrm{bol}}$ for a set of 63 QSOs with spectra in two or more datasets. These comparisons are shown in Fig. 1. The quality of the relative spectrophotometric calibration governs the rms scatter of $\approx 0.11$ dex in the independent measurements of $L_{\mathrm{bol}}$. The scatter in $M_{\mathrm{BH}}$ is a bit larger $(\approx 0.24 \mathrm{dex})$ 

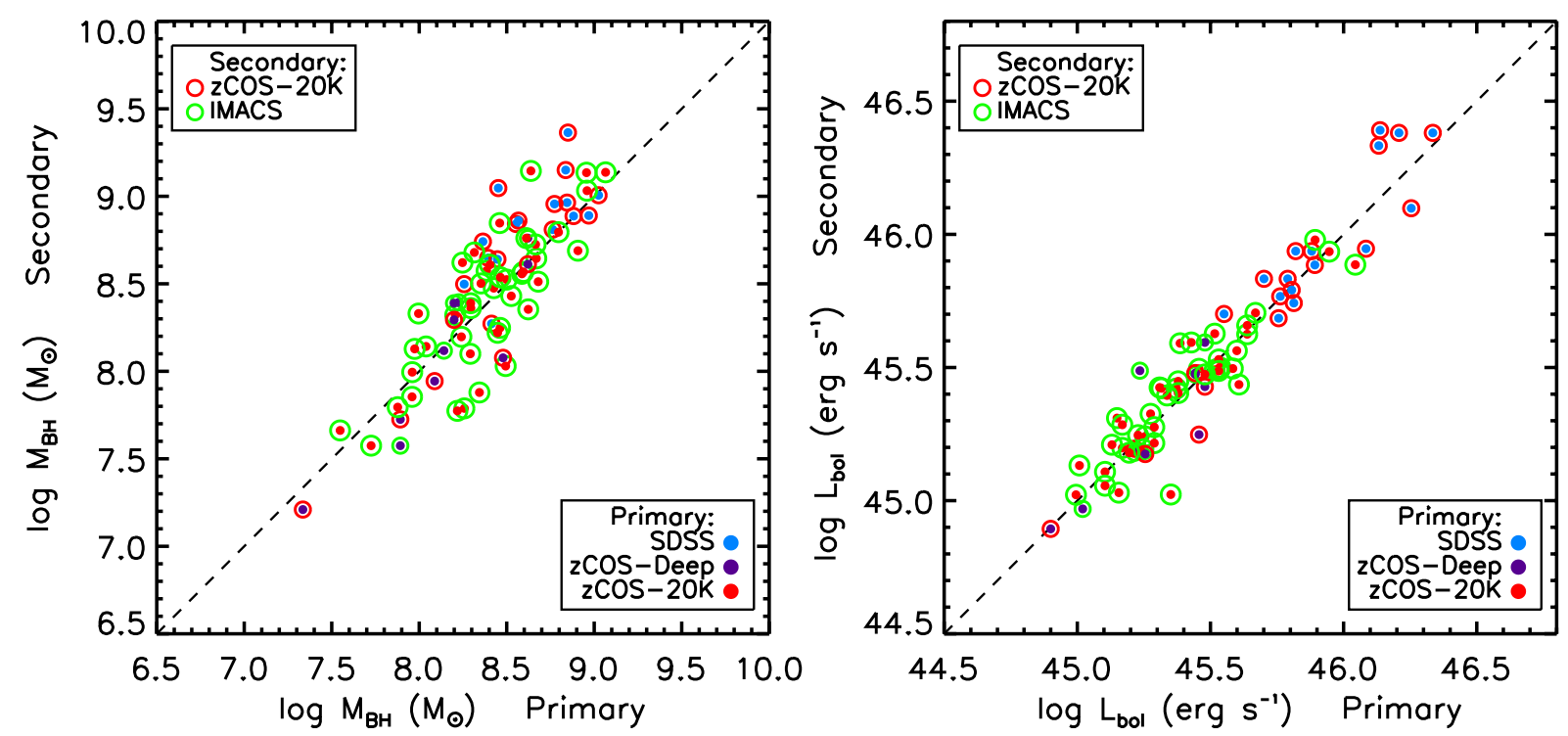

Fig. 1. Comparison of black hole mass $\left(M_{\mathrm{BH}}\right)$ estimates (left panel) and bolometric luminosity $\left(L_{\mathrm{bol}}\right)$ estimates (right panel) for a set of QSOs that have at least two spectra from different datasets. The estimate from the primary dataset is plotted on the $x$-axis and the estimate from the secondary dataset is plotted on the $y$-axis, where the hierarchy is based on preferences outlined in Sect. 4. The color of the solid core circle identifies the primary dataset, which is either SDSS (blue), zCOSMOS-Deep (purple), or zCOSMOS-Bright (red). The color of the outer circle identifies the secondary dataset, which may be zCOSMOS-Bright (red) or IMACS (green). The $L_{\mathrm{bol}}$ estimates, derived from direct measurements of the local continuum from the spectra, are very consistent between datasets. The $M_{\mathrm{BH}}$ estimates show more scatter and a small systematic variation about the $1: 1$ line.

and reflects the sensitivity of the broadline fitting technique to the $\mathrm{S} / \mathrm{N}$ of the spectra and the effects of fringing noise in the red ends of the zCOSMOS spectra. We also find a significant offset of $\approx 0.2$ dex between $M_{\mathrm{BH}}$ from SDSS and zCOSMOS spectra, towards higher masses from the second dataset - inspection suggests that this is due to fringing in the red part of the zCOSMOS spectra affecting the fits. For this reason, we default to using the SDSS fits for objects where an overlap exists between the two datasets. We adopt a conservative uncertainty of 0.3 dex in $M_{\mathrm{BH}}$ in further analysis, which takes into account the scatter and possible systematic offsets across the fits.

\subsection{FIR luminosities: detection, stacking, and measurement}

As a direct tracer of the FIR emission we concentrate on the mean luminosity $v L_{v}(60 \mu \mathrm{m})$, estimated at a rest-frame wavelength of $60 \mu \mathrm{m}$ (henceforth $L_{60}$ ). This choice is set by a wavelength long enough to avoid significant AGN contamination and short enough to be sampled by PACS $160 \mu \mathrm{m}$ observations even at the highest redshifts considered in this work.

We study trends of $L_{60}$ of BLAGNs binned in redshift and additionally in SMBH mass $\left(M_{\mathrm{BH}}\right)$, AGN bolometric luminosity $\left(L_{\text {bol }}\right)$, or SMBH specific accretion rate, expressed as the fraction of the Eddington luminosity $\left(\lambda_{\mathrm{E}}\right)$. Our Herschel/PACS data were used to measure $L_{60}$ for objects in a bin, in the manner detailed in Shao et al. (2010) and Santini et al. (2012). We briefly describe it here.

At each PACS band, a small fraction of sources $(\approx 10 \%-15 \%)$ are detected in both PACS bands. For these sources, $L_{60}$ is calculated using their individual redshifts and a log-linear interpolation of PACS fluxes. Of the remaining sources, some are detected in only one PACS band, while the majority are undetected in the FIR data. For this second group, we stacked at the optical positions of the AGNs on PACS residual maps using routines developed with the Béthermin et al. (2010)
FIR stacking libraries, from which we derive mean fluxes in both bands using PSF photometry. We then average the stacked fluxes with the fluxes of sources singly detected in either PACS band, weighting by the number of sources. This gives mean fluxes for the partially detected and undetected AGNs in both bands, from which we derive a mean $L_{60}$ using the median redshift of these sources. The final $60 \mu \mathrm{m}$ luminosity in each bin was computed by averaging over the linear luminosities of detections and nondetections, weighted by the number of sources. This procedure was only performed for bins with more than three sources in total.

Errors in the infrared luminosity are obtained by bootstrapping, in a fashion similar to that used in Shao et al. (2010). A set of sources equal to the number of sources per bin is randomly chosen 100 times among detections and non-detections (allowing repetitions), and $L_{60}$ is computed per each iteration. The standard deviation of the obtained $L_{60}$ values gives the error on the average $60 \mu \mathrm{m}$ luminosity in each bin. The error bars thus account for both measurement errors and the scatter in the population distribution.

\subsubsection{AGN contamination in the FIR}

Most of the AGNs studied in this work are relatively luminous systems, spanning the turnover in the AGN luminosity function (e.g., Marconi et al. 2004; Hopkins et al. 2007). Even if a fraction of their bolometric output is reprocessed by cold dust in their host galaxies, these AGNs could significantly alter, or even dominate, the FIR luminosity of their host galaxies. However, several studies of QSOs have shown that most of the dust reprocessed output in AGNs is in the form of hot dust emission, which peaks at mid-infrared wavelengths (Schweitzer et al. 2006; Netzer et al. 2007; Rafferty et al. 2011; Mullaney et al. 2011; Mor \& Netzer 2012; Rosario et al. 2012) and drops off steeply to the FIR. This implies that the AGN bolometric correction at a rest wavelength 

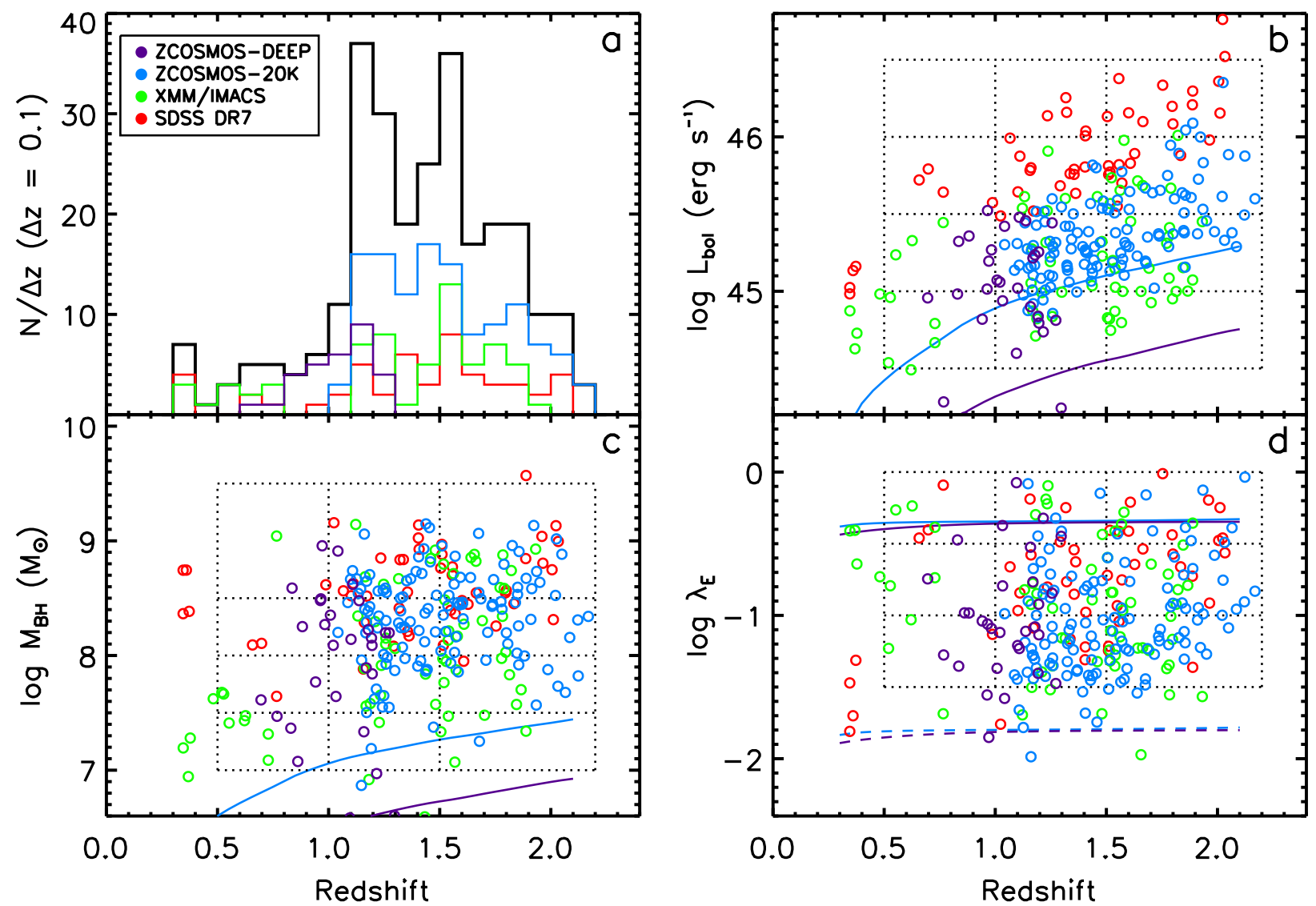

Fig. 2. Properties of the COSMOS QSO working sample (duplicates resolved). a) Redshift distribution of the sample: all QSOs (black) and for each individual dataset, colored according to the key in the upper-left corner. b) AGN bolometric luminosity against redshift. The solid lines show the limiting $L_{\mathrm{bol}}$ set by the $I_{A B}=22.5$ and $B_{A B}=25$ depths of the zCOSMOS Bright and Deep surveys, respectively. c) Black hole mass $\left(M_{\mathrm{BH}}\right)$ against redshift. The solid lines show the limiting $M_{\mathrm{BH}}$ set by the limiting magnitude of the zCOSMOS datasets for SMBHs with broad-line $F W H M=1500 \mathrm{~km} \mathrm{~s}^{-1}$. d) Eddington ratio $\left(\lambda_{\mathrm{E}}\right)$ against redshift. Lines show the $\lambda_{\mathrm{E}}$ limits set by the limiting magnitude of the zCOSMOS datasets, for two different broad-line FWHM: $1500 \mathrm{~km} \mathrm{~s}^{-1}$ (solid) and $8000 \mathrm{~km} \mathrm{~s}^{-1}$ (dashed). The points and limit lines in the all panels are colored by dataset according to the key in panel a). The bins used to divide the sample by redshift and $M_{\mathrm{BH}}, L_{\mathrm{bol}}$, and $\lambda_{\mathrm{E}}$ are shown as dotted lines in the respective panels. Objects in each binned subsample were stacked together in the Herschel/PACS maps to derive mean FIR luminosities for the bin.

of $60 \mu \mathrm{m} k_{60}\left(\equiv L_{\mathrm{bol}} / L_{60}\right)$ is $\gg 1$. Accounting for the diversity of empirically determined spectral energy distributions (SEDs) of AGNs (Netzer et al. 2007; Mullaney et al. 2011), and using the methodology described in Rosario et al. (2012), we estimate the bolometric correction to have the form

$\log k_{60}=1.65+0.2 \log L_{\mathrm{bol}, 46}$,

where $L_{\mathrm{bol}, 46}$ is $L_{\mathrm{bol}}$ in units of $10^{46} \mathrm{erg} \mathrm{s}^{-1}$. The expected scatter in $k_{60}$ is $\approx 0.3$ dex, due to intrinsic variation in the IR SED shapes of AGNs and from the real scatter in the local X-ray to MIR correlation used in Rosario et al. (2012) to connect IR to total AGN emission.

\section{Sample properties}

The redshift distribution of our BLAGN sample is shown in Fig. 2a. Most of the sample lies between $z=1$ and $z=2$ because the zCOSMOS AGN, the largest part of the sample, were specifically chosen to include the MgII line, which enters into the wavelength range of the zCOSMOS-Bright spectra at $z \sim 1$. The SDSS, IMACS, and zCOSMOS-Deep subsamples (the last covering bluer wavelengths than zCOSMOS-Bright) contribute to the tail of sources at lower redshifts.
For all the FIR stacking analyses in this work, we divide the AGNs into subsamples on the basis of redshift. We will use the following fiducial redshift bins: $0.5<z<1.0,1.0<z<1.5$, $1.5<z<2.2$, designated as the low, intermediate, and high redshift bins, respectively.

In the other three panels of Fig. 2, we plot the SMBH mass $\left(M_{\mathrm{BH}}\right)$, the AGN bolometric luminosity $\left(L_{\mathrm{bol}}\right)$, and the specific accretion rate or Eddington ratio $\left(\lambda_{\mathrm{E}}\right)$ against redshift. The bins in redshift, $M_{\mathrm{BH}}, L_{\mathrm{bol}}$, and $\lambda_{\mathrm{E}}$ used in the stacking analyses of Sect. 5 are shown as boxes with dotted outlines in the figure. These bins cover essentially all the AGN in our sample. Details of the binning scheme, including the number of objects in each bin, are listed in Table 1.

In Fig. 2, different colors are used to represent the different spectral datasets used in this work. In cases where the same object is observed in two different programs, we adopt one of the measurements following the hierarchy SDSS $>$ zCOSMOSDeep $>$ zCOSMOS-Bright $>$ IMACS (justified in Sect. 3.1).

Objects from the different spectral surveys occupy different and complementary areas in the space of BLAGN properties. The sharpest contrast is in $L_{\text {bol }}$, where the SDSS AGNs occupy the luminous end at all redshifts, while the zCOSMOS and IMACS AGNs fill in the sample to lower luminosities, providing a roughly uniform sampling of AGNs with 
Table 1. Mean rest-frame $60 \mu \mathrm{m}$ luminosities in bins of redshift and SMBH properties.

\begin{tabular}{lcccc}
\hline \hline Redshift bins & $0.3-0.5$ & $0.5-1.0$ & $1.0-1.5$ & $1.5-2.2$ \\
\hline All AGNs & $44.12-44.36(3 / 7)$ & $44.48-44.82(2 / 20)$ & $45.02-45.14(18 / 115)$ & $45.29-45.43(13 / 110)$ \\
\hline Bolometric luminosity $\left(\log L_{\text {bol }}\right)$ & & & & \\
\hline $44.5-45.0$ & - & $43.92-44.27(0 / 7)$ & $43.62-44.44(0 / 16)$ & $44.48-44.90(0 / 10)$ \\
$45.0-45.5$ & - & $44.65-45.02(1 / 8)$ & $44.97-45.17(10 / 66)$ & $45.06-45.31(4 / 43)$ \\
$45.5-46.0$ & - & $44.55-45.00(1 / 5)$ & $45.19-45.38(7 / 28)$ & $45.39-45.61(8 / 43)$ \\
$46.0-47.0$ & - & $-(0)$ & $44.25-44.94(1 / 5)$ & $45.42-45.62(1 / 14)$ \\
\hline Black hole mass $\left(\log M_{\mathrm{BH}}\right)$ & - & & \\
\hline $7.0-7.5$ & - & $44.23-45.12(1 / 5)$ & $45.06-45.82(1 / 4)$ & $44.93-45.29(0 / 6)$ \\
$7.5-8.0$ & - & $43.99-44.47(0 / 5)$ & $44.61-44.97(3 / 26)$ & $44.86-45.32(1 / 19)$ \\
$8.0-8.5$ & - & $44.30-44.81(1 / 6)$ & $44.90-45.16(3 / 41)$ & $45.01-45.22(4 / 42)$ \\
$8.5-9.5$ & - & $44.74-44.93(0 / 4)$ & $45.05-45.23(11 / 44)$ & $45.50-45.71(8 / 43)$ \\
\hline Eddington ratio $\left(\log \lambda_{\mathrm{E}}\right)$ & - & & \\
\hline$-1.5--1.0$ & - & $44.34-44.69(0 / 6)$ & $44.90-45.08(6 / 53)$ & $45.32-45.55(7 / 44)$ \\
$-1.0--0.5$ & - & $44.37-44.44(0 / 4)$ & $45.07-45.25(8 / 39)$ & $45.23-45.41(4 / 40)$ \\
$-0.5-0.0$ & - & $44.61-45.08(2 / 7)$ & $44.51-45.44(1 / 11)$ & $45.17-45.44(2 / 21)$ \\
\hline
\end{tabular}

Notes. Mean rest-frame $60 \mu \mathrm{m}$ luminosities are in units of $\log \mathrm{erg} \mathrm{s}^{-1}$. In parentheses at each entry: number of QSOs detected in PACS in a bin/total number of QSOs in a bin.

$L_{\text {bol }}>10^{44.5-45.0} \mathrm{erg} \mathrm{s}^{-1}$ over all redshifts. The SDSS AGNs are also typically at higher $M_{\mathrm{BH}}$ and higher $\lambda_{\mathrm{E}}$ than AGNs from the other surveys. The zCOSMOS-Deep AGNs occupy a fairly narrow range in redshift between $0.8<z<1.3$ and a range of $L_{\text {bol }}$ that is lower than AGNs from the other samples. The parent zCOSMOS-Deep spectral survey has a color-preselection which chooses galaxies with $z>1.5$, which is in contrast to the actual redshifts of the AGNs from the survey. This is likely because the zCOSMOS-Deep AGNs have optical SEDs that are dominated by AGN light, while the color-preselection is only valid for galaxy-dominated SEDs.

The flux limits set by the noise properties of the spectral datasets or the magnitude limits of the various surveys introduce redshift-dependent luminosity limits to our sample. For example, the limits of the two zCOSMOS surveys are shown as solid lines in Fig. 2b. The IMACS spectra go to fainter fluxes than zCOSMOS-Bright at $z>1.5$, but still describe a fluxlimited subsample. The limits shown here have been calculated for $\operatorname{MgII} 12800$, the primary broad line used for mass measurements in this work. At $z \lesssim 0.7$, only $\mathrm{H} \beta$ is visible in most spectra as MgII enters the UV atmospheric cutoff. However, since our method is cross-calibrated between these two lines, the limits shown here are essentially identical irrespective of the line used for the $M_{\mathrm{BH}}$ measurement.

There is an equivalent lower limit to $M_{\mathrm{BH}}$, since lowmass SMBHs produce narrower broad-lines, with $F W H M<$ $1500 \mathrm{~km} \mathrm{~s}^{-1}$, and will not be easily identified as BLAGNs in spectral datasets. This, combined with the dependence of $M_{\mathrm{BH}}$ on $L_{\text {bol }}$, sets an approximate lower envelope to the SMBH masses in our sample, shown with the solid lines in Panel c. There is no formal upper-limit to the $M_{\mathrm{BH}}$ distribution, but SMBHs with very broad lines $\left(F W H M>10^{5} \mathrm{~km} \mathrm{~s}^{-1}\right)$ are never found (Trump et al. 2009b). The combination of the lower limits to $L_{\mathrm{bol}}$ and $M_{\mathrm{BH}}$ sets a lower limit to $\lambda_{\mathrm{E}}$ which is FWHM dependent, as shown for the zCOSMOS spectra in Panel d. Both zCOSMOS Deep and Bright limits overlap in this plot. Interestingly, the limit is almost flat with redshift. In other words, our sample selects objects with the same range in specific accretion rates across all redshifts in this study.
It is worthwhile noting here that the AGNs in the low redshift bin do not include many objects with high $M_{\mathrm{BH}}$ when compared to the two higher redshift subsamples. This may be purely stochastic or due to Malmquist bias, but it does lead to some complexity in the interpretation of SFR trends in Sect. 5.

\subsection{Selection effects in the $M_{\mathrm{BH}}-L_{\text {bol }}$ plane}

In Fig. 3, we plot the AGN bolometric luminosity against the SMBH mass for the QSO sample, with separate panels for each fiducial redshift bin. Lines of constant Eddington ratio are shown as dotted lines and labeled accordingly. The distribution of objects in this diagram has a characteristic form. In each bin, there is a rough lower limit to $L_{\text {bol }}$, below which essentially no objects are found. This is an observational bias set by the flux limits in our sample (see above). Besides this, one can also notice that the range in $M_{\mathrm{BH}}$ displayed by BLAGNs is also a function of $L_{\mathrm{bol}}$. In particular, at low bolometric luminosities, one can notice a tail of low-mass AGNs, which are typically absent at higher AGN luminosities. This selection effect is driven mostly by the steep drop in the incidence of AGN with increasing SMBH specific accretion rate (e.g., Schulze \& Wisotzki 2010). Objects with low SMBH masses are selected only if they have $\lambda_{\mathrm{E}}$ high enough to lie above the luminosity threshold set by the observational limits. However, the space density of such rapidly accreting systems is low at all redshifts, and hence most of the low-mass SMBHs in our sample are found close to the $L_{\text {bol }}$ limit in all three bins. At higher SMBH masses, even systems with low specific accretion rates satisfy the luminosity threshold. As relatively more AGNs are found at low $\lambda_{\mathrm{E}}$ than high $\lambda_{\mathrm{E}}$, the observed specific accretion rate distribution of our sample will change across $M_{\mathrm{BH}}$. When considering AGN with low-mass SMBHs, our sample contains a large fraction of rapidly accreting systems $\left(\lambda_{\mathrm{E}} \sim 1\right)$, while at high $M_{\mathrm{BH}}$, one finds a majority of slowly accreting AGN $\left(\lambda_{\mathrm{E}} \sim 0.03\right)$. These patterns mirror those found among the brighter SDSS QSO population (Steinhardt \& Elvis 2010). Since $M_{\mathrm{BH}}$ correlates with stellar mass which, in turn, correlates with SFR, these selection effects must be kept in mind when interpreting 


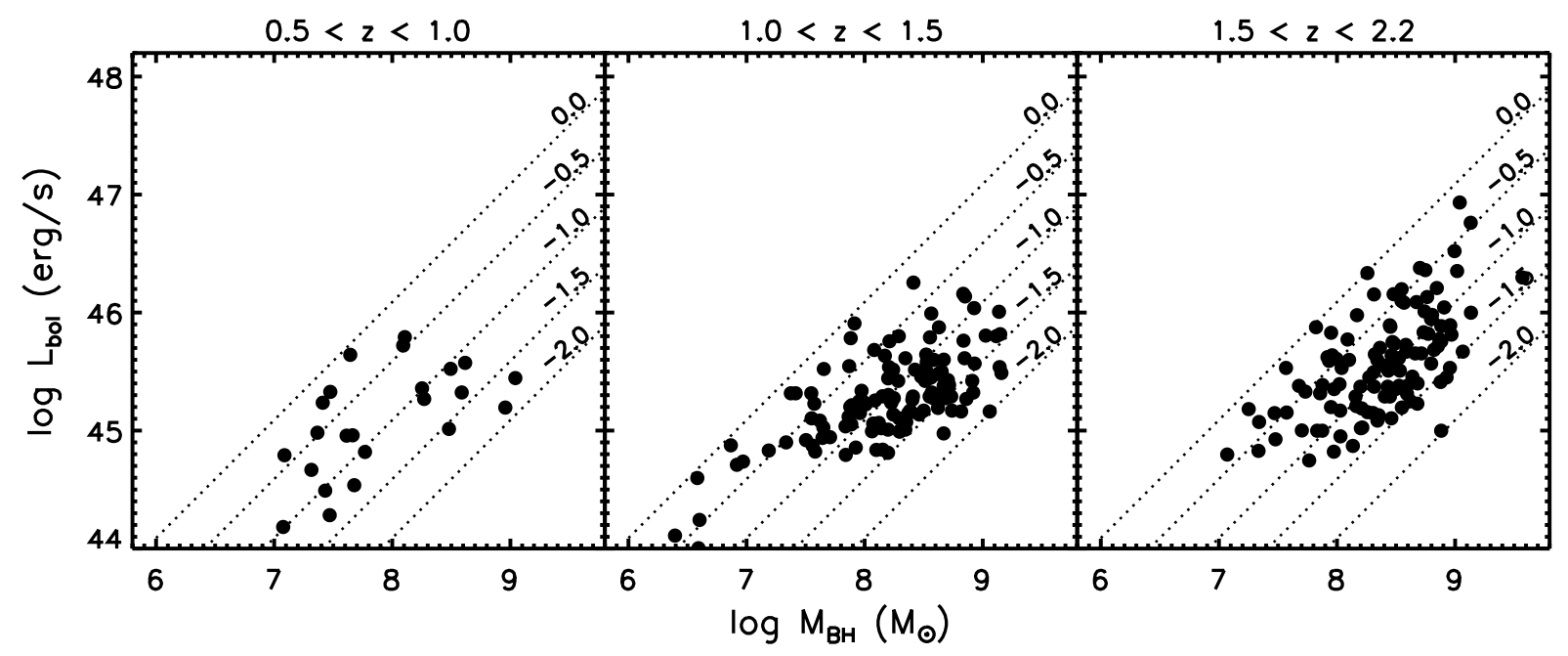

Fig. 3. Black hole mass $\left(M_{\mathrm{BH}}\right)$ vs. bolometric luminosity $\left(L_{\mathrm{bol}}\right)$ for QSOs in three redshift bins in the COSMOS field. Lines of constant Eddington ratio $\left(\lambda_{\mathrm{E}}\right)$ are shown as dotted sloped lines and are labeled with $\log \lambda_{\mathrm{E}}$.

trends between SFR and various BLAGN properties, as we do in Sect. 5 .

\section{Results: The mean SFR of BLAGNs}

\subsection{Trends with redshift: a comparison to X-ray AGNs}

The BLAGN sample in this work is a subset of the population of luminous AGNs in the COSMOS field, specifically those with unobscured lines of sight to the broad-line region around the accreting black holes. By and large, they are also a subset of the X-ray AGN population in that field, since only a small fraction of the BLAGNs are not detected in the XMM-COSMOS survey (Sect. 2.3). In an earlier study (Rosario et al. 2012), we constrained the mean SF properties, as measured by $L_{60}$, of a larger and more complete set of AGNs from XMM-COSMOS selected on the basis of their X-ray emission (Cappelluti et al. 2009; Brusa et al. 2010). Here, we consider the redshift evolution of $L_{60}$ of our BLAGNs. This serves two purposes: it allows us to set a baseline for the SF properties of the QSO population selected in COSMOS across redshift, and to compare the typical SF properties of BLAGNs with the larger X-ray selected population, which can be instructive in revealing potential differences between the host galaxies of QSOs and other AGNs, as well as highlighting selection biases in BLAGN samples.

In Fig. 4 , we plot the mean $L_{60}$ of all BLAGNs in our sample binned by redshift. In addition to the fiducial redshift bins listed in Sect. 4, we include an additional low redshift bin at $0.3<z<0.5$ for a longer redshift baseline. The $x$-axis error bars show the range in redshift that contain $80 \%$ of all objects in a bin contributing to the mean $L_{60}$ measurement, while the $y$-axis errors come from bootstrap resampling of the stacked sample. In the figure, we also show the evolution with redshift of the mean $L_{60}$ for X-ray AGNs from Rosario et al. (2012) in two bins in hard-band (2-10 keV) X-ray luminosity: $10^{43}-10^{44} \mathrm{erg} \mathrm{s}^{-1}$ and $10^{44}-10^{45} \mathrm{erg} \mathrm{s}^{-1}$. These correspond roughly to the luminosities of local powerful Seyferts and QSOs respectively. A detailed discussion of the offsets in $L_{60}$ between these lines and their evolution with redshift can be found in Rosario et al. (2012).

At low redshifts, the $L_{60}$ of the BLAGNs are consistent with that of X-ray AGNs in the luminous Seyfert range, while at $z>$ 1 , their $L_{60}$ are comparable with the more luminous X-ray AGNs. At first glance, one might mistakenly attribute the difference in

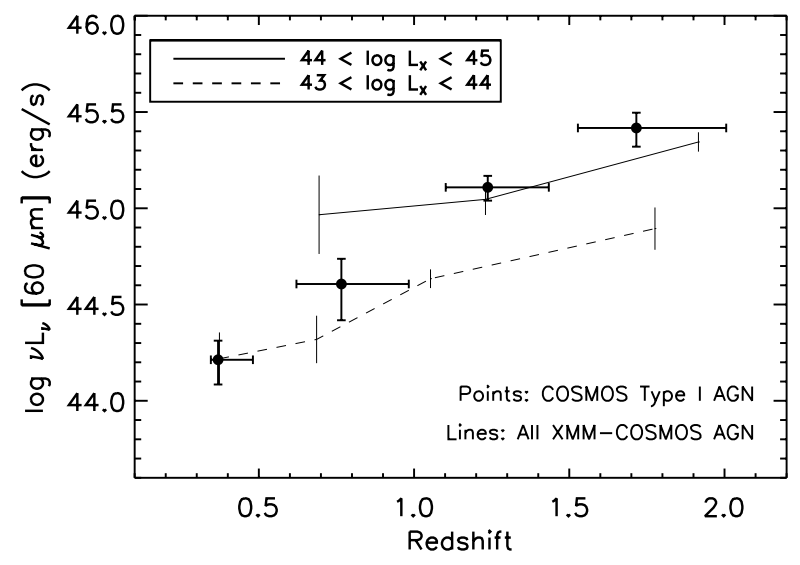

Fig. 4. Mean $60 \mu \mathrm{m}$ luminosity $L_{60}$ of QSOs in COSMOS as a function of redshift, shown as solid dots with error bars. The $x$-axis error bars show the range in redshift that encompasses $80 \%$ of the sample in the respective redshift bin. The solid and dashed lines show the mean trends for AGNs from the XMM-COSMOS survey (Brusa et al. 2010) in two bins in instrinsic 2-10 keV X-ray luminosity. Vertical error bars placed at intervals on these lines show the $1 \sigma$ scatter in these trends.

the typical FIR luminosities of low and high redshift BLAGNs as a sign that the mean SFR of BLAGNs evolves more rapidly with redshift than the mean SFR of the general population of X-ray AGNs. However, in reality, the difference in redshift evolution is primarily governed by the change in the typical luminosity of BLAGNs with redshift.

In Fig. 5, we compare the $\mathrm{X}$-ray luminosities of $\mathrm{X}$-ray detected BLAGNs with the full population of X-ray AGNs from XMM-COSMOS. At $z<1$, BLAGNs are more luminous than the typical X-ray AGN, but at higher redshifts, both sets span similar ranges in X-ray luminosity. Nevertheless, the typical $\mathrm{X}$-ray luminosity of BLAGNs increases with redshift, from around $10^{43.5}$ in the $0.3<z<0.5$ bin to $10^{44.3}$ at $1.5<z<2.2$. This behavior is set purely by the particular flux limits of the COSMOS spectral datasets. Considering Figs. 4 and 5 together, we see that the steeper increase in $L_{60}$ with redshift of the BLAGNs is simply due to the change in the typical AGN luminosity of the population. The mean SFR of BLAGNs is consistent with the mean SFR of similarly luminous X-ray AGNs. 


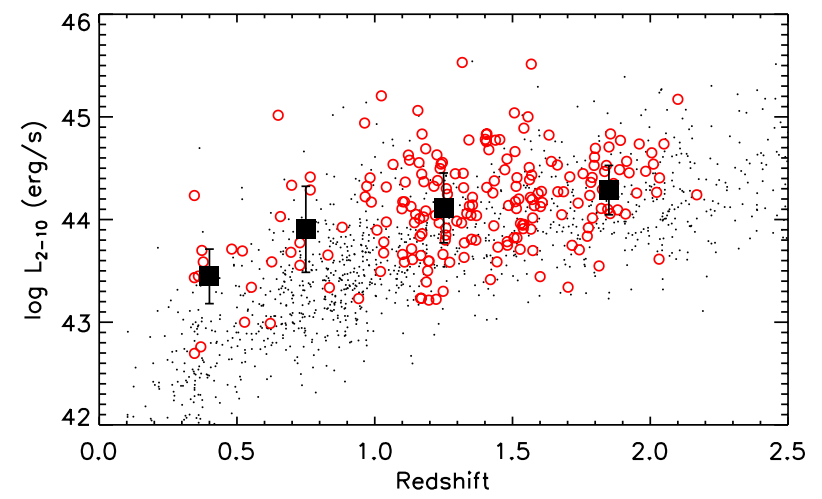

Fig. 5. 2-10 keV luminosities of X-ray AGNs (small black points) and X-ray detected QSOs (large red open points) over redshift, based on photometry from the XMM-COSMOS survey (Brusa et al. 2010). The mean X-ray luminosity (in log units) of QSOs in our nominal redshift bins are plotted as large black square points, with the error bars showing the median absolute deviation of the data points from the mean. QSOs are typically more luminous than most X-ray AGNs at low redshifts, but span a similar range in X-ray luminosities as XMM-COSMOS AGNs at $z>1$. Despite this, there is an increase in the mean X-ray luminosity with redshift for the QSOs in our sample.

\subsection{Trends with AGN bolometric luminosity}

In Rosario et al. (2012), we explored the relationships between $\mathrm{SF}$ and the luminous output of X-ray AGNs, uncovering a relationship between $L_{60}$ and $L_{\text {bol }}$ in luminous AGNs at $z<1$. This increase suggested an elevated role for mergers at high AGN luminosities, as predicted by various evolutionary models for SMBH growth (e.g., Hopkins et al. 2008). Here we place our BLAGN sample in the context of our earlier study to test if the trends uncovered among all X-ray AGN are also evident in the unobscured luminous AGN studied in this work.

In Fig. 6, we plot mean $L_{60}$ of the BLAGNs, measured from stacks in bins of $L_{\mathrm{bol}}$ and redshift. The AGNs were divided into four bins in $\log L_{\mathrm{bol}}(44.5-45.0,45.0-45.5,45.5-46.0$, and 46.046.5) as well as the fiducial redshift bins. Additionally, only those AGNs with $\log M_{\mathrm{BH}}$ between 7.0 and 9.5 were considered for stacking for consistency with other binning schemes discussed below. The data points in the figure show the mean $L_{60}$ for all bins with enough objects for a valid measurement $(N>3)$. The $y$-axis error bars come from bootstrapping into the stacked sample, while $x$-axis error bars that show the range in $L_{\text {bol }}$ that encompasses $80 \%$ of the subsample in each bin. Included as well in the figure are lightly shaded regions which show the empirical trends between $L_{\text {bol }}$ and $L_{60}$ uncovered for X-ray AGNs from Rosario et al. (2012). These trends suggest a correlation between AGN luminosity and SFR at $z<1$, but a flatter relationship at higher redshift.

The BLAGNs display a different behavior in the $L_{\mathrm{bol}}-L_{60}$ plane compared to X-ray AGNs. The FIR luminosities of the AGNs in the lowest $L_{\text {bol }}$ bin are systematically lower than with those of the X-ray AGNs at the same bolometric luminosity. In addition, the trends of the BLAGN measurements in all three redshift bins show a characteristic shape, with a sharp increase in $L_{60}$ with $L_{\text {bol }}$ at low AGN bolometric luminosities that flattens or drops at high AGN luminosities in a redshift dependent manner. At low $L_{\text {bol }}$, optical BLAGN appear to show a lower SFR than X-ray AGN of the same bolometric luminosity, while at intermediate $L_{\text {bol }}\left(\sim 10^{45.5} \mathrm{erg} \mathrm{s}^{-1}\right)$ they appear to show an elevated mean SFR. A first comparison of the different trends in the $L_{\mathrm{bol}}-$ $L_{60}$ plane may lead one to conclude that luminous unobscured

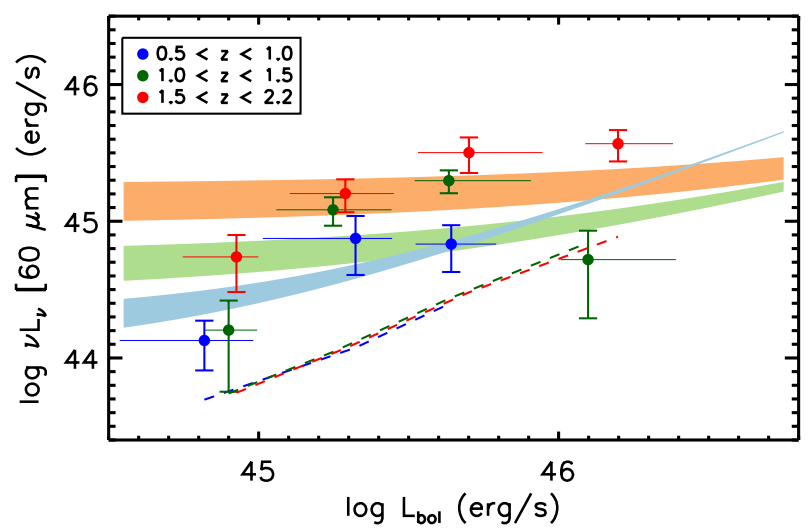

Fig. 6. Mean $L_{60}$ of QSOs as a function of AGN bolometric luminosity $L_{\mathrm{bol}}$, shown as colored dots with error bars. AGNs in the $0.5<z<1.0$, $1.0<z<1.5$, and $1.5<z<2.2$ redshift bins are represented by blue, green, and red colors, respectively. The $x$-axis error bars show the range in $L_{\mathrm{bol}}$ that encompasses $80 \%$ of the sample in the corresponding bolometric luminosity bin. Dashed lines show the expected contribution to the FIR luminosity from pure AGN-heated dust (Eq. (1)). The lightly shaded colored polygons show the mean $L_{60}$ trends for X-ray AGNs from the fits in Rosario et al. (2012). The difference in the shape of the trends between X-ray AGNs and QSOs can be attributed to selection biases in the sample (see Sect. 5.2 for details).

AGNs show different host SF signatures compared to a broader $\mathrm{X}$-ray selected sample. However, a closer examination of the selection effects inherent in such BLAGN samples suggests a different interpretation.

From Fig. 3, we see that more luminous AGN are, on average, associated with more massive SMBHs. Since SMBH mass is correlated with the stellar mass of the host galaxy which, in turn, is correlated with SFR, the lower luminosity BLAGNs in our sample contain a larger fraction of low-mass host galaxies that will bring down their average SFR. At high $L_{\mathrm{bol}}$, the characteristic flattening seen at low and intermediate redshift AGNs can be interpreted in one of two ways: a) the most luminous AGN are responsible for quenching SF in their hosts, or, alternatively; b) such AGN are typically found in high-mass galaxies (owing to the selection effects outlined above) which, for various reasons not yet well understood, contain a higher quenched fraction at all redshifts. As we show in Sect. 6.1, a model that does not require widespread AGN-driven quenching in luminous BLAGNs can explain these trends quite well.

Using Eq. (1), we estimate the AGN contribution to the $60 \mu \mathrm{m}$ luminosity of the BLAGNs in our sample in the different bins in $L_{\text {bol }}$ and redshift. For these estimates, we also include a random term to capture the scatter in $k_{60}$, since our measured mean $L_{60}$, a linear combination, is disproportionately affected by upward logarithmic scatter than downward scatter. The expected AGN contribution to $L_{60}$, evaluated from 1000 random estimates, is shown as dashed lines in Fig. 6. Except for the lowest luminosity bin at low redshift and some of the highest luminosity bins, AGN contamination does not strongly influence trends we see in this diagram, especially those seen at $z \sim 2$. It is also unlikely to influence any of the other trends we present later in this work. Accounting for it may, however, strengthen the turnover that we see at high AGN luminosities in the low and intermediate redshift bins in Fig. 6. In Sect. 6, we develop the discussion of AGN contamination and its role in the interpretation of these trends. 


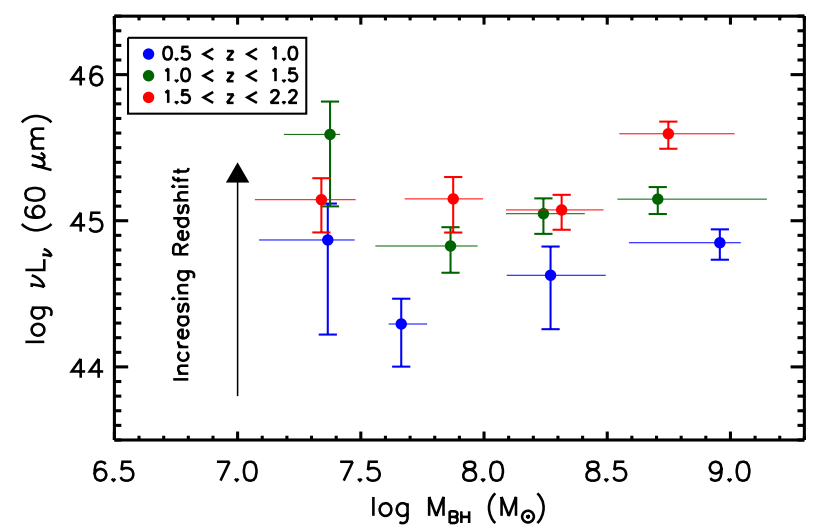

Fig. 7. Mean $L_{60}$ of BLAGNs as a function of SMBH mass $M_{\mathrm{BH}}$, shown as colored dots with error bars. AGNs in the $0.5<z<1.0,1.0<z<$ 1.5 , and $1.5<z<2.2$ redshift bins are represented by blue, green, and red colors, respectively. The $x$-axis error bars show the range in $M_{\mathrm{BH}}$ that encompasses $80 \%$ of the sample in the corresponding mass bin.

\subsection{Trends with black hole mass}

Black hole mass $\left(M_{\mathrm{BH}}\right)$ is a fundamental property of an SMBH and determines the maximum amount of energy that can be derived from the accreting system. SMBH demographic studies have revealed a strong correlation between the mass of a black hole and the total stellar content of the host spheroid in local galaxies (the $M_{\mathrm{BH}}-\sigma$ relation, Häring \& Rix 2004; Sani et al. 2011), the form of which may evolve with redshift (Jahnke et al. 2009; Merloni et al. 2010; Bennert et al. 2011; but see Schulze \& Wisotzki 2011). Since the spheroid mass is related to the total mass of the galaxy, correlations between $M_{\mathrm{BH}}$ and $M_{*}$ are expected and are indeed found (e.g., Sani et al. 2011).

The SFR of galaxies is known to correlate with stellar mass along a ridgeline in SFR- $M_{*}$ space popularly called the mass sequence of galaxies (Noeske et al. 2007; Elbaz et al. 2007; Daddi et al. 2007b; Rodighiero et al. 2011; Whitaker et al. 2012). When combined with the $M_{\mathrm{BH}}-\sigma$ relation, the SFR mass sequence predicts a relationship of the mean SFR of BLAGNs with $M_{\mathrm{BH}}$, since more massive SMBHs are found in more massive hosts which, in turn, have a larger SFR. Do we find such a relationship among our BLAGN sample?

In Fig. 7, we plot the mean $L_{60}$ vs. $M_{\mathrm{BH}}$, binning the AGNs in redshift and SMBH mass following the scheme in Table 1. In the low redshift bin, there is a significant increase in the mean SFR between the hosts of $10^{7.5}<M_{\mathrm{BH}}<10^{8.0} M_{\odot}$ black holes and the hosts of $10^{8.5}<M_{\mathrm{BH}}<10^{9.5} M_{\odot}$ black holes. The mean SFR of systems with $10^{8.0}<M_{\mathrm{BH}}<10^{8.5} M_{\odot}$ SMBHs lies in between and spans the difference, within the errors. A similar trend, with some scatter, is seen at higher redshifts, with the increase extending to the highest masses probed. In contrast, there is a hint that the mean FIR luminosities of the lowest mass SMBHs, in the $10^{7.0}<M_{\mathrm{BH}}<10^{7.5} M_{\odot}$ bins, do not follow the trend exhibited by more massive SMBHs, but, in the low and intermediate redshift bins, have systematically higher $L_{60}$ than expected. This may indicate that the lowest mass SMBHs are in hosts with elevated specific SFRs than the rest of the SMBH population. We caution, however, that the enhancement is marginal at best and is subject to the highly biased nature of the low-mass SMBH population in our sample (Sect. 4.1).

To constrain the trend between the median $M_{\mathrm{BH}}$ (in $\log M_{\odot}$ ) and the mean $L_{60}$ (in $\log \mathrm{erg} \mathrm{s}^{-1}$ ), we fit a simple straight line to the stacked points, excluding the pathological lowest mass bins

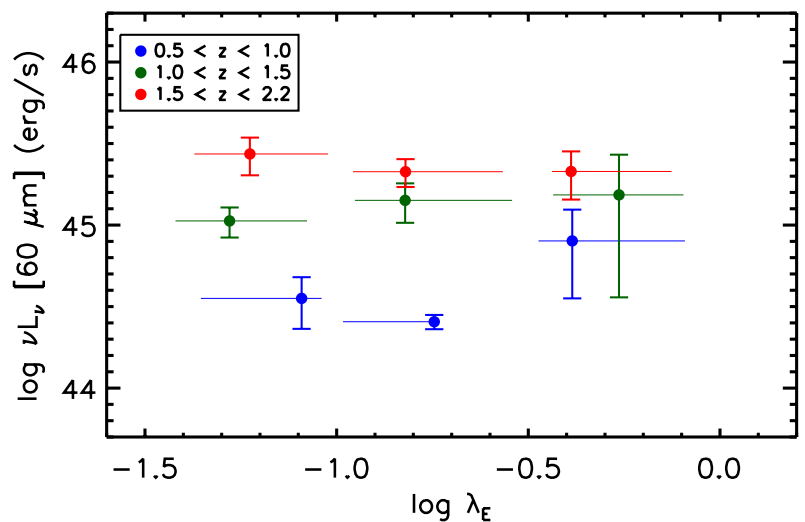

Fig. 8. Mean $L_{60}$ of BLAGNs as a function of AGN specific accretion rate (the Eddington ratio $\lambda_{\mathrm{E}}$ ), shown as colored dots with error bars. AGNs in the $0.5<z<1.0,1.0<z<1.5$, and $1.5<z<2.2$ redshift bins are represented by blue, green, and red colors, respectively. The $x$-axis error bars show the range in $\lambda_{\mathrm{E}}$ that encompasses $80 \%$ of the sample in the corresponding Eddington ratio bin.

and including the errors in mean $L_{60}$. The slopes of the trends from the fits are $0.71 \pm 0.47,0.34 \pm 0.20$, and $0.74 \pm 0.25$ in the low, intermediate, and high redshift bins respectively. Within the uncertainties, the slopes are consistent with being constant with redshift, with a weighted average value from all three redshift bins of $\approx 0.5$. This is similar to, but a bit shallower than, the expected value of 0.7 at $z=1$ if $S F R \propto M_{*}{ }^{0.57}$ (Whitaker et al. 2012) and $M_{*} \propto M_{\mathrm{BH}}{ }^{0.79}$ (Sani et al. 2011). The uncertainties in our measurements prevent us from making any conclusions about possible evolution in the relationship between $M_{\mathrm{BH}}$ and SFR, which could arise from, for example, a change in the slope of the $M_{\mathrm{BH}}-M_{*}$ relationship with cosmic time.

\subsection{Trends with Eddington ratio}

We now turn to the study of relationships between the SFR of BLAGN hosts and the specific accretion rate of the $\operatorname{SMBH}\left(\lambda_{\mathrm{E}}\right)$. In the context of close co-evolution between galaxies and their SMBHs, many models predict strong evolution in the stellar content of an AGN host galaxy while the SMBH is growing at a fast rate. Therefore, one expects a higher than average SFR for AGN hosts with fast growing SMBHs, while galaxies with slowly growing SMBHs will show a slower growth rate or relatively normal SFR for their stellar mass. There is some evidence of this among local Seyfert 1 AGNs (Sani et al. 2010).

In Fig. 8, we show measurements of the mean FIR luminosities for our BLAGN sample in three bins of redshift and $\lambda_{\mathrm{E}}$ (as listed in Table 1). In general, there are no strong systematic trends between $\lambda_{\mathrm{E}}$ and $L_{60}$. Among the two higher redshift subsamples, $L_{60}$ is consistently flat between $\lambda_{\mathrm{E}}=0.03$ and $\lambda_{\mathrm{E}}=1$, while in the low redshift bin, AGNs with $\lambda_{\mathrm{E}}>0.3$ exhibit an increase in $L_{60}$ more rapidly (by a factor of 3 ) over slower accreting objects, which is significant at the level of $\approx 2 \sigma$.

How would the biases inherent in our sample influence these trends? As discussed in Sect. 4.1 and from Fig. 3, the most highly accreting objects at all redshifts include a larger proportion of low-mass SMBHs. Low $M_{\mathrm{BH}}$ systems are found in low-mass host galaxies, which, in turn, have lower typical SFR. Therefore, the mean SFR of our subsample of highly accreting SMBHs may be depressed somewhat by the greater fraction of low-mass host galaxies compared to AGNs at lower $\lambda_{\mathrm{E}}$. If a positive correlation does exist between SFR (or $L_{60}$ ) and $\lambda_{\mathrm{E}}$, it will be flattened by 
the inclusion of these low-mass galaxies at high $\lambda_{\mathrm{E}}$. However, a more detailed understanding of the effects of selection biases requires modeling, which we pursue in the following section.

\section{Discussion}

We explored two alternative approaches to mitigate the effects of the sample biases on the observed trends between $L_{60}$ and various Type-I AGN properties. One was to apply a lower limit in SMBH mass of $M_{\mathrm{BH}}>10^{7.8} M_{\odot}$ to our sample. As one can see in Fig. 3, this serves to remove the long tail to low $M_{\mathrm{BH}}$, particularly at $z>1$. The resulting reduced sample is less sensitive to $M_{\mathrm{BH}}$-based selection effects. However, this approach also discards any information carried among the lower mass SMBHs, while the smaller sample size leads to insufficient numbers of objects for a good PACS stacking signal in the important high $\lambda_{\mathrm{E}}$ bins. Therefore, we turned to a second approach. We developed a heuristic empirically-constrained model for the AGN host galaxy population, to enable a prediction of mean $L_{60}$ and its trends directly from our measurements of $M_{\mathrm{BH}}$, based on known scaling relationships between SMBH mass, $M_{*}$, and SFR. By default, this subsumes any biases in the sample into the predicted trends. Here, we present the details of this approach, show how selection effects in the sample influence (and restrict) the results of our mean FIR study, and discuss what we can learn about the BLAGN host galaxy population from the trends.

\subsection{The baseline model}

In order to make an estimate of the mean $L_{60}$ for our BLAGNs, we require some input on the nature of AGN emission, their host galaxies, and the evolution of their scaling relations. We make the following assumptions: a) AGN hosts are subsets of normal star-forming galaxies, i.e., they are not preferentially in special populations such as starbursts or major mergers; b) AGN hosts lie on the mass sequence of normal star-forming galaxies; c) the $M_{\mathrm{BH}}-M_{*}$ relationship remains constant with redshift; and d) the FIR bolometric correction (Eq. (1)) of AGNs does not evolve with redshift. These assumptions define a baseline model for the BLAGN population. If there are substantial deviations between the data and our predictions, this will serve as a test of the assumptions built into the baseline model.

A prediction of the mean $L_{60}$ for our BLAGNs is developed as follows. For all objects in a bin of redshift and/or either $M_{\mathrm{BH}}$, $L_{\text {bol }}$, or $\lambda_{\mathrm{E}}$, we estimate stellar masses from SMBH masses by inverting Eq. (8) of Sani et al. (2011). From $M_{*}$, we derive SFRs using the mass sequence relation from Whitaker et al. (2012). We convert the SFRs to $60 \mu \mathrm{m}$ luminosities using the standard calibration from Kennicutt (1998), taking a mean ratio of 0.5 between $L_{60}$ and the integrated $8-1000 \mu \mathrm{m}$ luminosity, based on the FIR SED libraries of Chary \& Elbaz (2001). We use a Monte Carlo bootstrap approach to propagate the scatter in these relations, $\sigma\left(M_{\mathrm{BH}} / M_{*}\right)=0.35 \mathrm{dex}, \sigma\left(M_{*} / S F R\right)=0.3 \mathrm{dex}$, and $\sigma\left(S F R / L_{60}\right)=0.18$ dex, by randomly varying the assumed relationships around their central trend by these $\sigma$. In addition, we include a small enhancement to the $L_{60}$ to account for the AGN hot-dust contribution at $60 \mu \mathrm{m}$, which is calculated from $L_{\mathrm{bol}}$ using Eq. (1), with a scatter of 0.3 dex. From 1000 iterations, we arrive at a mean predicted SFR for the ensemble of objects in each bin as well as a prediction for the uncertainty on the mean arising from the intrinsic scatter of AGN host galaxy properties in our model.
In Fig. 9, we compare predictions of mean $L_{60}$ against our $L_{60}$ measurements. The shaded regions indicate the range in mean $L_{60}$ expected from the baseline model following our Monte-Carlo treatment of scatter. The $x$-axis values of the model regions are pinned to the actual median value of $M_{\mathrm{BH}}, L_{\mathrm{bol}}$, and $\lambda_{\mathrm{E}}$ in any given redshift bin, since the input to the model are the empirical measurements for the very objects that belong to each bin. In general, the model matches the observed data points quite well, both in the redshift evolution of $L_{60}$, the trends with SMBH parameters, and also in the scatter about these trends. The biggest deviations between the data and the model arise in bins with small numbers of objects $(N \lesssim 6)$; these bins can be severely affected by stochastic effects which are not accounted for by the bootstrap error estimates. The uncertainties on the mean $L_{60}$, while large in these bins, are probably still underestimated.

The PACS stacks indicate a positive correlation between $L_{60}$ and $M_{\mathrm{BH}}$. The predicted slope of this trend in the baseline model is determined almost completely by the slope of the SF mass sequence and is a good representation of the actual trends seen in the data, indicating that BLAGN hosts also lie along the SF sequence to $z=2.2$. As suggested in Sect. 5.2, the rather unusual shapes of the $L_{60}-L_{\mathrm{bol}}$ trends are now shown to be driven almost completely by selection effects in the $M_{\mathrm{BH}}-L_{\text {bol }}$ plane. The baseline model assumes no direct connection between $L_{60}$ and the accretion luminosity of the AGN (except for a small, generally negligible, component from AGN heated dust), yet the predicted trends show a characteristic slope that arises only because the low $L_{\text {bol }}$ bins contain a larger fraction of low-mass AGN host galaxies that bring down the mean $L_{60}$. Similarly, while the baseline model does not adopt any explicit relationship between the SMBH Eddington ratio and SFR, it predicts a flat or falling trend of $L_{60}$ with $\lambda_{\mathrm{E}}$ since the population of the most rapidly accreting black holes in our sample also includes a greater fraction of low-mass systems.

The good agreement between the baseline model and the stacked $L_{60}$ measurements suggests that such a simple model can actually be a good representation of the population of BLAGN host galaxies. Taken at face value, this agreement implies that BLAGN hosts are not in obvious strong starbursts, since this population is expected to lie well above the SF mass sequence. Instead, most QSO host galaxies are apparently in normal massive star-forming galaxies. However, we caution against too liberal an interpretation, since this conclusion depends on the validity of the assumptions that go into the baseline model. Firstly, the model assumes that all host galaxies lie on the SF mass sequence, i.e., AGNs hosts are all forming stars. It is known, however, that a substantial fraction of massive galaxies lie in quiescent hosts that we have not accounted for in the model. Optical imaging of QSO hosts suggest that most show signs of on-going SF (e.g., Jahnke et al. 2004; Trump et al. 2013), while Herschel-based studies of lower luminosity X-ray AGNs show that they are preferentially found in SF galaxies (Rosario et al. 2013). Taken together, the assumption that QSOs are in SF hosts may be reasonably valid. Furthermore, objects with very weak or absent SF do not contribute significantly to the stacked flux, meaning that possible quiescent AGN hosts in our sample are deweighted in the mean measured $L_{60}$. Another wrinkle arises because the adopted $M_{\mathrm{BH}}-M_{*}$ relationship (Eq. (8) of Sani et al. 2011) is valid only for the bulge stellar mass, while, in the fashion of Merloni et al. (2010), we use it with the total galaxy stellar mass. In addition, we are also aware that SMBH scaling laws may vary at the highest mass end $\left(M_{\mathrm{BH}}>10^{9} M_{\odot}\right)$ and lowest mass end $\left(M_{\mathrm{BH}}<10^{7} M_{\odot}\right)$ 
Baseline Model
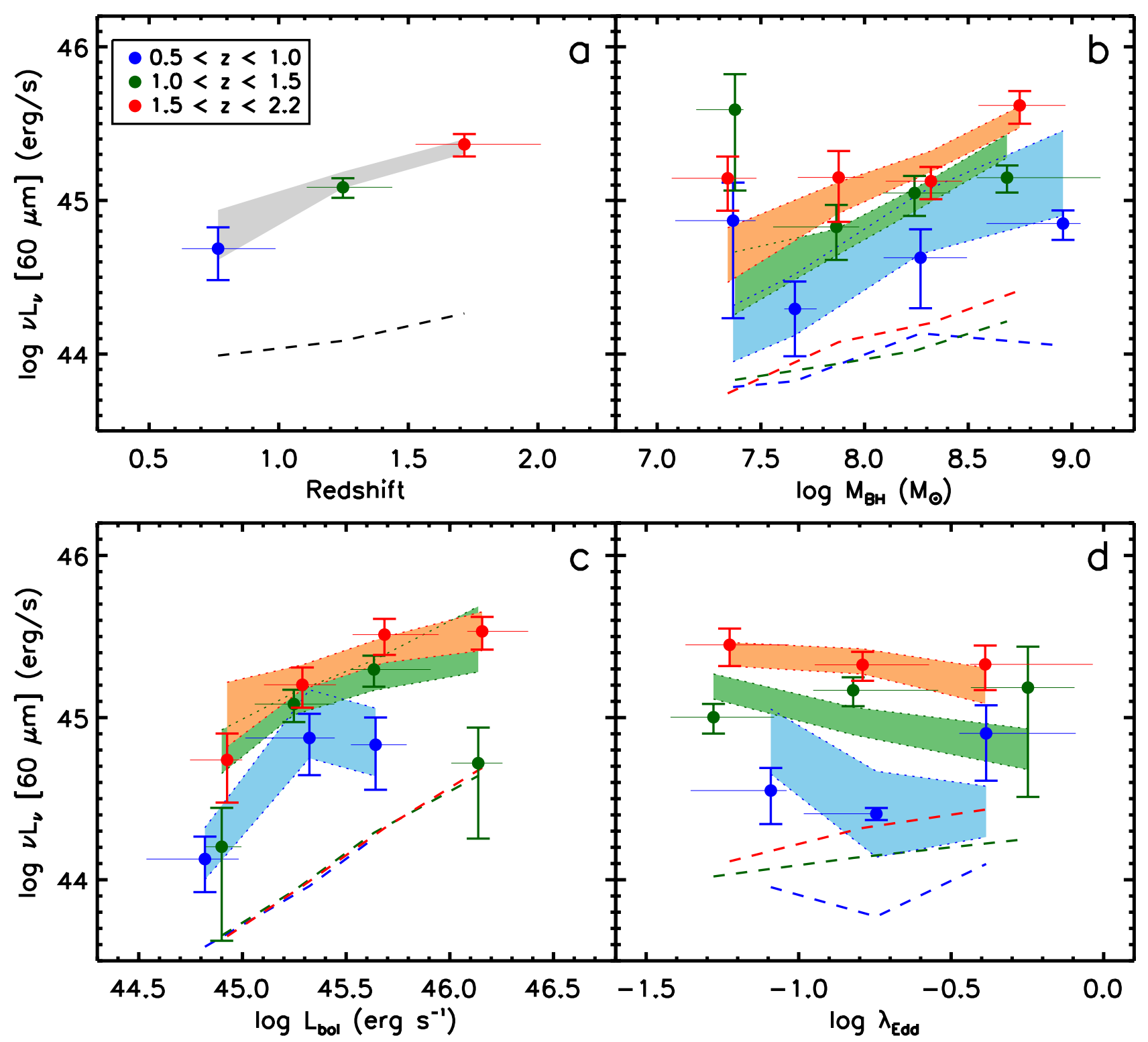

Fig. 9. Comparison between the measured mean $L_{60}$ and the predictions of a baseline model that, through a simple bootstrap analysis, takes into account some of the selection effects built into the QSO sample (see Sect. 6.1 for details). AGNs in the $0.5<z<1.0,1.0<z<1.5$, and $1.5<z<2.2$ redshift bins are represented by blue, green, and red colors, respectively. The four panels show measurements as colored dots with error bars, in a) bins of redshift only, b) bins of $M_{\mathrm{BH}}$ and redshift, c) bins of $L_{\mathrm{bol}}$ and redshift, and d) bins of $\lambda_{\mathrm{E}}$ and redshift. The $x$-axis error bars are not uncertainties, but a range in the abscissa that encompasses $80 \%$ of the sample in the corresponding bin. Dashed lines show the expected contribution to the FIR luminosity from pure AGN-heated dust. The shaded polygons show the baseline model predictions for the trends: gray for panel a) and blue/green/red in the other three panels representing the three redshift bins according to the key in a). The $y$-axis width of the polygons gives the $1 \sigma$ uncertainty on the mean model trends, determined by the intrinsic scatter of the model QSO host population and the size of each subsample. In all panels, the form of the trends and the uncertainties on the measurements are reproduced reasonably well by the baseline model, especially in bins with more than ten objects.

(e.g., Graham 2012; van den Bosch et al. 2012; Graham \& Scott 2013), but this is unlikely to influence our results, since most of our AGNs have masses within these extremes.

The sizable uncertainties on the measurements prevent a finer investigation into the parameter space of the models. Given the primarily empirical nature of this work, we restrict our study of models to a few simple tests in which we explore the performance of the models when we vary the offset of the AGNs from the SF mass sequence, or include possible evolution in the $M_{\mathrm{BH}}-M_{*}$ scaling relation.

\subsection{Varying the offset from the SF mass sequence}

Co-evolutionary scenarios that link AGN activity with bursts in SF predict that AGNs should be found in host galaxies that lie above the SF mass sequence. We perform a simple test of these scenarios by adding a redshift-independent offset to the SFR in our baseline model and then compare the goodness of fit of the altered model to that of the baseline model (i.e., the model with normal star-forming AGN hosts). As a measure of the goodness of fit, we calculate the $\chi^{2}$ statistic of the model predictions with 


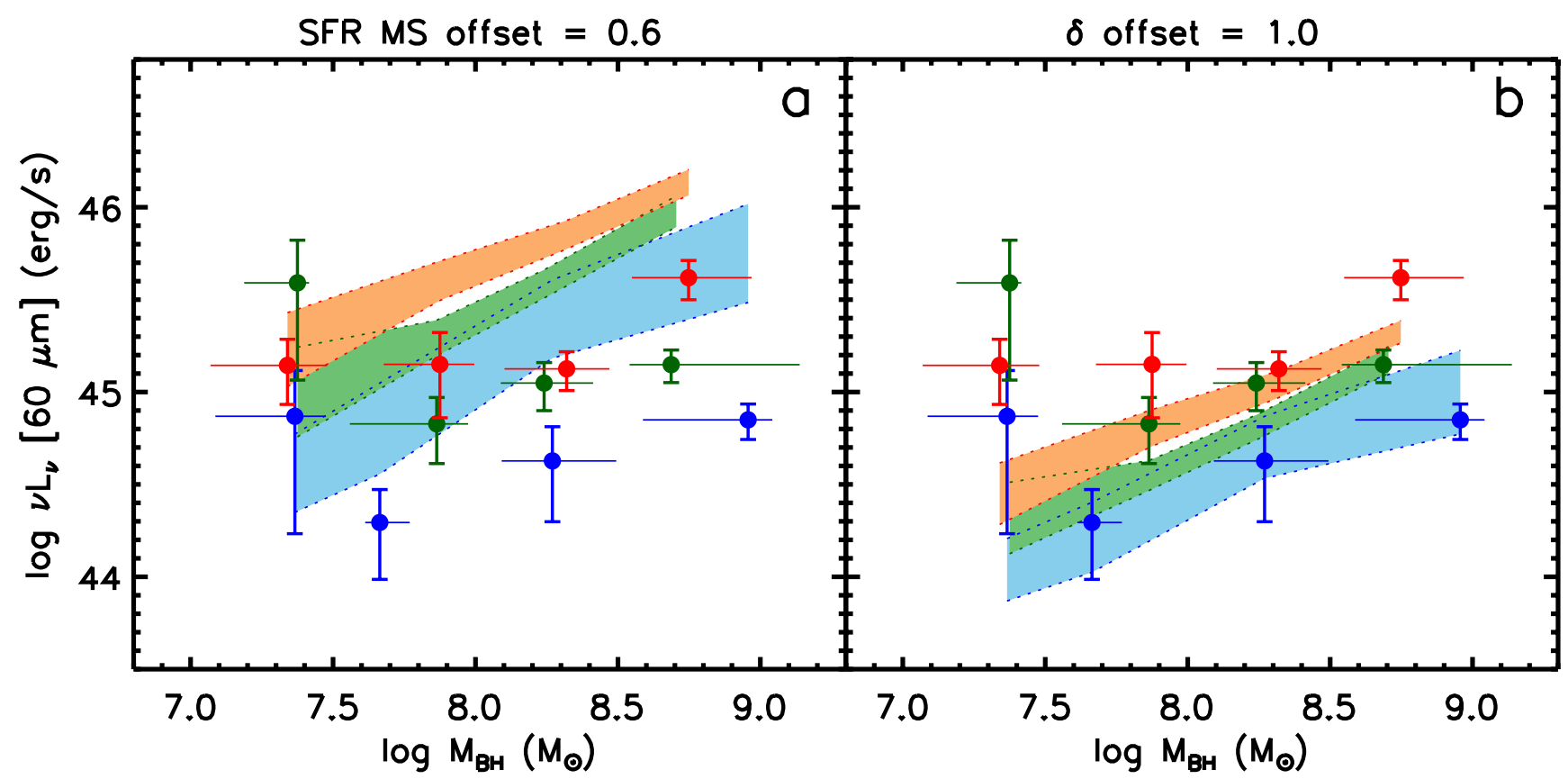

Fig. 10. Comparison between the measured mean $L_{60}$ in bins of $M_{\mathrm{BH}}$ and the predictions of two simple alternative models for BLAGN host properties. a) All BLAGNs are assumed to lie in starbursts which have at a mean positive offset of 0.6 dex in SFR from the SF Mass Sequence, or b) the $M_{\mathrm{BH}}-M_{*}$ relation evolves with redshift according to Eq. (2) with $\delta=1.0$. The measurements are shown as colored points with error bars. AGNs in the $0.5<z<1.0,1.0<z<1.5$, and $1.5<z<2.2$ redshift bins are represented by blue, green, and red colors, respectively. The $x$-axis error bars show the range in the $M_{\mathrm{BH}}$ that encompasses $80 \%$ of the sample in the corresponding bin. Neither set of parameters chosen here fit the data as well as the "baseline model" (Sect. 6.1). While model a) performs very poorly in fitting the data, model b) is marginally acceptable, though smaller values of $\delta$ are preferred.

respect to the stacked measurements in bins of $M_{\mathrm{BH}}$ and redshift bins (Fig. 7). The bootstrap errors on the measurements are used to weigh the data in the estimation of the statistic. Better fitting models yield lower values of $\chi^{2}$. We caution, however, that the $\chi^{2}$ discussed below are meant to allow a qualitative judgement of variation arising from model parameters. One should not use them for robust statistical estimates of confidence intervals or for significance testing.

For the baseline model, we calculate a $\chi^{2}=3.1$. If AGN hosts are in a starburst phase offset in SFR by +0.6 dex from the mass sequence (Rodighiero et al. 2011; Sargent et al. 2012), the $\chi^{2}$ increases to 7.5 (left panel of Fig. 10). This sharp increase clearly disfavors a scenario where all BLAGNs are in strong starbursts. Even a minor SFR offset over the mass sequence results in an increased $\chi^{2}$ (to 3.4 for a +0.1 dex offset, for example). Indeed, the $\chi^{2}$ reaches a minimum with negative offsets, placing QSOs very slightly below the mass sequence $(-0.05$ dex gives a $\left.\chi^{2}=3.0\right)$. Since there is some debate as to the exact form for the mass sequence (Noeske et al. 2007; Elbaz et al. 2007; Daddi et al. 2007b; Wuyts et al. 2011; Whitaker et al. 2012), it may be that the baseline model is a slightly incorrect representation of the true galaxy population, and certainly a -0.05 dex offset is within most uncertainties in the sequence. Another explanation arises if we consider evolution in the $M_{\mathrm{BH}}-M_{*}$ relation from the canonical form we adopted in the baseline model.

\subsection{Varying the normalization of the $M_{\mathrm{BH}}-M_{*}$ relation}

Recent studies of the evolution of SMBH scaling laws suggest an increase in the normalization of the $M_{\mathrm{BH}}-M_{*}$ with redshift (e.g., Decarli et al. 2010; Merloni et al. 2010; Trakhtenbrot \& Netzer 2010; Bennert et al. 2011; Targett et al. 2012), which can be parameterized in the following fashion:

$\Delta M_{\mathrm{BH}} / M_{*}=\delta \log (1+z)$,

where $\Delta M_{\mathrm{BH}} / M_{*}$ is the offset from the local $M_{\mathrm{BH}}-M_{*}$ relation (Sani et al. 2011). These various studies have calibrated the slope of the redshift term $\delta=0.5-2.0$, with substantial uncertainty. A positive $\delta$ results in a smaller $M_{*}$ for a given $M_{\mathrm{BH}}$ at higher redshifts, which will produce a smaller mean SFR and lower $L_{60}$ compared to the baseline model. However, reviews of the biases inherent in studies of the evolution of SMBH scaling laws (Schulze \& Wisotzki 2011; Salviander \& Shields 2013) suggest that $\delta$ may be quite unconstrained by empirical studies and even $\delta=0$ could be consistent with such studies. By including a term from Eq. (2) to the $M_{\mathrm{BH}}-M_{*}$ relation in the baseline model, we can test for the effects of changing SMBH relations. We find that a small positive value of $\delta$ does improve the performance of the model, but only slightly $\left(\delta=0.25\right.$ yields a minimum $\left.\chi^{2}=3.0\right)$. Stronger redshift evolution leads again to larger $\chi^{2}$, for example, at $\delta=0.5$, the low end of the range from direct studies, $\chi^{2}=3.1$, while for $\delta=2.0, \chi^{2}=4.8$. In the right panel of Fig. 10, we show the performance of the model with $\delta=1.0\left(\chi^{2}=3.4\right)$. Clearly, our measurements are most consistent with very mild to no redshift evolution in the $M_{\mathrm{BH}}-M_{*}$ relation.

\subsection{The star forming properties of QSO host galaxies}

We have shown that the mean SFR of QSOs with nuclear bolometric luminosities in the range $L_{\mathrm{bol}}=10^{44.5-46.5}$ can be described quite well by our baseline model, in which QSO hosts are normal SF galaxies which lie on the mass sequence. We find that a slight evolution in the $M_{\mathrm{BH}}-M_{*}$ relation with redshift is supported by the modeling of our measurements. We 
also show that the scenario in which QSO hosts contain starbursts (i.e., have a positive offset from the SF mass sequence) and the scenario where $M_{\mathrm{BH}}$ increasingly precedes $M_{*}$ with redshift (Eq. (2) with a positive $\delta$ ) have opposite effects on the modeled SFR $-M_{\mathrm{BH}}$ relationship. Therefore, a situation in which QSO hosts, in truth, lie increasingly among starbursts at higher redshifts may be offset by a positive evolution in the $M_{\mathrm{BH}}-M_{*}$ relationship. Satisfying a pure starburst scenario (as defined by Rodighiero et al. 2011; Sargent et al. 2012) is highly unlikely, since it would require a very high $\delta$ which is not supported by observations. At present, our measurements cannot distinguish between the simple baseline model and a scenario with modest $\delta$ evolution coupled with a moderate starburst fraction. Having said this, the simplest scenario, based on the assumptions of the baseline model, works quite well and a more complex model will have to bring together firm complementary evidence to support it over the basic baseline model.

\section{Conclusions}

Combining diverse spectroscopic datasets from the COSMOS extragalactic survey, we compile one of the largest samples of moderate luminosity QSOs to $z \sim 2$ with deep FIR data from the PEP survey and uniform measurements of SMBH properties. We extensively characterize the sample and highlight important selection effects which play a role in understanding its properties. The QSO database is used to explore the relationships between SFR and $M_{\mathrm{BH}}, L_{\mathrm{bol}}$, and SMBH specific accretion rate $\lambda_{\mathrm{E}}$. After accounting for selection effects using a Monte Carlo bootstrapping procedure, we show that the SFR trends of QSOs out to $z \sim 2$ are most consistent with a simple model where their hosts are galaxies that lie on the SF mass sequence. Scenarios where all QSO hosts are in strong starbursts are inconsistent with our measurements. The typical SFRs of galaxies hosting the fastest growing black holes are not significantly enhanced over systems with slower growing black holes. Our modeling also indicates that the redshift evolution of SMBH-host scaling relationships may be rather mild. Taken at face value, our results suggest that QSOs at the luminosities that dominate the volume-averaged SMBH growth at $z=2$ lie in fairly normal star-forming host galaxies, which set important constraints on models of AGN-galaxy co-evolution and the processes that influence SMBH scaling laws.

Acknowledgements. PACS has been developed by a consortium of institutes led by MPE (Germany) and including UVIE (Austria); KU Leuven, CSL, IMEC (Belgium); CEA, LAM (France); MPIA (Germany); INAF-IFSI/ OAA/OAP/OAT, LENS, SISSA (Italy); IAC (Spain). This development has been supported by the funding agencies BMVIT (Austria), ESA-PRODEX (Belgium), CEA/CNES (France), DLR (Germany), ASI/INAF (Italy), and CICYT/MCYT (Spain). This research has made use of the NASA/IPAC Infrared Science Archive, which is operated by the Jet Propulsion Laboratory, California Institute of Technology, under contract with the NASA. zCOSMOS is based on observations made with ESO Telescopes at the La Silla and Paranal Observatories under programme ID 175.A-0839.

\section{References}

Abazajian, K. N., Adelman-McCarthy, J. K., Agüeros, M. A., et al. 2009, ApJS, 182,543

Alexander, D. M., Bauer, F. E., Brandt, W. N., et al. 2011, ApJ, 738, 44 Aller, M. C., \& Richstone, D. O. 2007, ApJ, 665, 120

Bahcall, J. N., Kirhakos, S., Saxe, D. H., \& Schneider, D. P. 1997, ApJ, 479, 642 Bennert, N., Canalizo, G., Jungwiert, B., et al. 2008, ApJ, 677, 846

Bennert, V. N., Auger, M. W., Treu, T., Woo, J.-H., \& Malkan, M. A. 2011, ApJ, 742,107
Béthermin, M., Dole, H., Beelen, A., \& Aussel, H. 2010, VizieR Online Data Catalog, J/A+A/512/A78

Bonfield, D. G., Jarvis, M. J., Hardcastle, M. J., et al. 2011, MNRAS, 416, 13

Brusa, M., Civano, F., Comastri, A., et al. 2010, ApJ, 716, 348

Capak, P., Aussel, H., Ajiki, M., et al. 2007, ApJS, 172, 99

Cappelluti, N., Brusa, M., Hasinger, G., et al. 2009, A\&A, 497, 635

Chabrier, G. 2003, PASP, 115, 763

Chary, R., \& Elbaz, D. 2001, ApJ, 556, 562

Ciotti, L., \& Ostriker, J. P. 2007, ApJ, 665, 1038

Coppin, K. E. K., Swinbank, A. M., Neri, R., et al. 2008, MNRAS, 389, 45

Daddi, E., Alexander, D. M., Dickinson, M., et al. 2007a, ApJ, 670, 173

Daddi, E., Dickinson, M., Morrison, G., et al. 2007b, ApJ, 670, 156

Dai, Y. S., Bergeron, J., Elvis, M., et al. 2012, ApJ, 753, 33

Decarli, R., Falomo, R., Treves, A., et al. 2010, MNRAS, 402, 2453

Donley, J. L., Rieke, G. H., Pérez-González, P. G., Rigby, J. R., \& AlonsoHerrero, A. 2007, ApJ, 660, 167

Dunlop, J. S., McLure, R. J., Kukula, M. J., et al. 2003, MNRAS, 340, 1095

Elbaz, D., Daddi, E., Le Borgne, D., et al. 2007, A\&A, 468, 33

Fiore, F., Grazian, A., Santini, P., et al. 2008, ApJ, 672, 94

Fiore, F., Puccetti, S., Brusa, M., et al. 2009, ApJ, 693, 447

Gilli, R., Comastri, A., \& Hasinger, G. 2007, A\&A, 463, 79

Graham, A. W. 2012, ApJ, 746, 113

Graham, A. W., \& Driver, S. P. 2007, ApJ, 655, 77

Graham, A. W., \& Scott, N. 2013, ApJ, 764, 151

Granato, G. L., De Zotti, G., Silva, L., Bressan, A., \& Danese, L. 2004, ApJ, 600,580

Guyon, O., Sanders, D. B., \& Stockton, A. 2006, ApJS, 166, 89

Häring, N., \& Rix, H.-W. 2004, ApJ, 604, L89

Hes, R., Barthel, P. D., \& Fosbury, R. A. E. 1993, Nature, 362, 326

Ho, L. C. 2005, ApJ, 629, 680

Hopkins, P. F., Richards, G. T., \& Hernquist, L. 2007, ApJ, 654, 731

Hopkins, P. F., Hernquist, L., Cox, T. J., \& Kereš, D. 2008, ApJS, 175, 356

Jahnke, K., Sánchez, S. F., Wisotzki, L., et al. 2004, ApJ, 614, 568

Jahnke, K., Bongiorno, A., Brusa, M., et al. 2009, ApJ, 706, L215

Kalfountzou, E., Jarvis, M. J., Bonfield, D. G., \& Hardcastle, M. J. 2012, MNRAS, 427, 2401

Kennicutt, Jr., R. C. 1998, ARA\&A, 36, 189

Kukula, M. J., Dunlop, J. S., McLure, R. J., et al. 2001, MNRAS, 326, 1533

Le Floc'h, E., Aussel, H., Ilbert, O., et al. 2009, ApJ, 703, 222

Lilly, S. J., Le Fèvre, O., Renzini, A., et al. 2007, ApJS, 172, 70

Lilly, S. J., Le Brun, V., Maier, C., et al. 2009, ApJS, 184, 218

Lutz, D., Sturm, E., Tacconi, L. J., et al. 2008, ApJ, 684, 853

Lutz, D., Mainieri, V., Rafferty, D., et al. 2010, ApJ, 712, 1287

Lutz, D., Poglitsch, A., Altieri, B., et al. 2011, A\&A, 532, A90

Magorrian, J., Tremaine, S., Richstone, D., et al. 1998, AJ, 115, 2285

Mainieri, V., Bongiorno, A., Merloni, A., et al. 2011, A\&A, 535, A80

Marconi, A., Risaliti, G., Gilli, R., et al. 2004, MNRAS, 351, 169

Martínez-Sansigre, A., Rawlings, S., Lacy, M., et al. 2005, Nature, 436, 666

Merloni, A., Bongiorno, A., Bolzonella, M., et al. 2010, ApJ, 708, 137

Mor, R., \& Netzer, H. 2012, MNRAS, 420, 526

Mullaney, J. R., Alexander, D. M., Goulding, A. D., \& Hickox, R. C. 2011, MNRAS, 414, 1082

Netzer, H., Lutz, D., Schweitzer, M., et al. 2007, ApJ, 666, 806

Noeske, K. G., Weiner, B. J., Faber, S. M., et al. 2007, ApJ, 660, L43

Omont, A., Beelen, A., Bertoldi, F., et al. 2003, A\&A, 398, 857

Page, M. J., Stevens, J. A., Ivison, R. J., \& Carrera, F. J. 2004, ApJ, 611, L85

Poglitsch, A., Waelkens, C., Geis, N., et al. 2010, A\&A, 518, L2

Polletta, M. d. C., Wilkes, B. J., Siana, B., et al. 2006, ApJ, 642, 673

Priddey, R. S., Isaak, K. G., McMahon, R. G., \& Omont, A. 2003, MNRAS, 339, 1183

Rafferty, D. A., Brandt, W. N., Alexander, D. M., et al. 2011, ApJ, 742, 3

Reyes, R., Zakamska, N. L., Strauss, M. A., et al. 2008, AJ, 136, 2373

Richards, G. T., Lacy, M., Storrie-Lombardi, L. J., et al. 2006, ApJS, 166, 470

Rodighiero, G., Daddi, E., Baronchelli, I., et al. 2011, ApJ, 739, L40

Rosario, D. J., Santini, P., Lutz, D., et al. 2012, A\&A, 545, A45

Rosario, D. J., Santini, P., Lutz, D., et al. 2013, ApJ, 771, 63

Salviander, S., \& Shields, G. A. 2013, ApJ, 764, 80

Sanders, D. B., Soifer, B. T., Elias, J. H., et al. 1988, ApJ, 325, 74

Sani, E., Lutz, D., Risaliti, G., et al. 2010, MNRAS, 403, 1246

Sani, E., Marconi, A., Hunt, L. K., \& Risaliti, G. 2011, MNRAS, 413, 1479

Santini, P., Rosario, D. J., Shao, L., et al. 2012, A\&A, 540, A109

Sargent, M. T., Béthermin, M., Daddi, E., \& Elbaz, D. 2012, ApJ, 747, L31

Schulze, A., \& Wisotzki, L. 2010, A\&A, 516, A87

Schulze, A., \& Wisotzki, L. 2011, A\&A, 535, A87

Schweitzer, M., Lutz, D., Sturm, E., et al. 2006, ApJ, 649, 79

Scoville, N., Aussel, H., Brusa, M., et al. 2007, ApJS, 172, 1

Serjeant, S., \& Hatziminaoglou, E. 2009, MNRAS, 397, 265 
A\&A 560, A72 (2013)

Serjeant, S., Bertoldi, F., Blain, A. W., et al. 2010, A\&A, 518, L7 Shao, L., Lutz, D., Nordon, R., et al. 2010, A\&A, 518, L26

Shen, Y., \& Kelly, B. C. 2012, ApJ, 746, 169

Shi, Y., Rieke, G. H., Ogle, P., Jiang, L., \& Diamond-Stanic, A. M. 2009, ApJ, 703,1107

Silverman, J. D., Lamareille, F., Maier, C., et al. 2009, ApJ, 696, 396

Steinhardt, C. L., \& Elvis, M. 2010, MNRAS, 402, 2637

Sturm, E., Hasinger, G., Lehmann, I., et al. 2006, ApJ, 642, 81

Targett, T. A., Dunlop, J. S., \& McLure, R. J. 2012, MNRAS, 420, 3621

Trakhtenbrot, B., \& Netzer, H. 2010, MNRAS, 406, L35

Trakhtenbrot, B., \& Netzer, H. 2012, MNRAS, 427, 3081

Tremaine, S., Gebhardt, K., Bender, R., et al. 2002, ApJ, 574, 740

Trump, J. R., Impey, C. D., McCarthy, P. J., et al. 2007, ApJS, 172, 383

Trump, J. R., Impey, C. D., Elvis, M., et al. 2009a, ApJ, 696, 1195
Trump, J. R., Impey, C. D., Kelly, B. C., et al. 2009b, ApJ, 700, 49 Trump, J. R., Hsu, A. D., Fang, J. J., et al. 2013, ApJ, 763, 133

van den Bosch, R. C. E., Gebhardt, K., Gültekin, K., et al. 2012, Nature, 491, 729

Veilleux, S., Kim, D.-C., Rupke, D. S. N., et al. 2009, ApJ, 701, 587

Vignali, C., Brandt, W. N., Fan, X., et al. 2001, AJ, 122, 2143

Walter, F., Carilli, C., Bertoldi, F., et al. 2004, ApJ, 615, L17

Walter, F., Riechers, D., Cox, P., et al. 2009, Nature, 457, 699

Wang, R., Carilli, C. L., Wagg, J., et al. 2008, ApJ, 687, 848

Wang, R., Carilli, C. L., Neri, R., et al. 2010, ApJ, 714, 699

Wang, R., Wagg, J., Carilli, C. L., et al. 2013, ApJ, 773, 44

Whitaker, K. E., van Dokkum, P. G., Brammer, G., \& Franx, M. 2012, ApJ, 754, L29

Wuyts, S., Förster Schreiber, N. M., van der Wel, A., et al. 2011, ApJ, 742, 96 